\title{
Holographic phase diagram of quark-gluon plasma formed in heavy-ion collisions
}

\author{
I.Ya. Aref'eva, ${ }^{a}$ A.A. Bagrov ${ }^{a, b}$ and E.O. Pozdeeva ${ }^{c}$ \\ ${ }^{a}$ Steklov Mathematical Institute, \\ Gubkin St. 8, 119991, Moscow, Russia \\ ${ }^{b}$ Instituut Lorentz, Leiden University, \\ Niels Bohrweg 2, 2300 RA Leiden, Netherlands \\ ${ }^{c}$ Skobeltsyn Institute of Nuclear Physics, Lomonosov Moscow State University, \\ Leninskie Gory, GSP-1, 119991, Moscow, Russia \\ E-mail: arefeva@mi.ras.ru, bagrov@lorentz.leidenuniv.nl, \\ pozdeeva@www-hep.sinp.msu.ru
}

ABSTRACT: We use a holographic dual model for the heavy-ion collision to obtain the phase diagram of the quark-gluon plasma (QGP) formed at a very early stage just after the collision. In this dual model, colliding ions are described by the charged gravitational shock waves. Points on the phase diagram correspond to the QGP or hadronic matter with given temperatures and chemical potentials. The phase of the QGP in dual terms is related to the case where the collision of shock waves leads to the formation of a trapped surface. Hadronic matter and other confined states correspond to the absence of a trapped surface after collision. In the dual language, the multiplicity of the ion collision process is estimated as the area of the trapped surface. We show that a nonzero chemical potential reduces the multiplicity. To plot the phase diagram, we use two different dual models of colliding ions, the pointlike and the wall shock waves, and find that the results agree qualitatively.

KEYWORDS: Holography and quark-gluon plasmas, Gauge-gravity correspondence

ARXIV EPRINT: 1201.6542 


\section{Contents}

1 Introduction $\quad 1$

2 Set up $\quad 4$

2.1 Dual conjectures 4

2.1.1 Black holes and the AdS/CFT correspondence for a strongly coupled QGP 4

2.1.2 The chemical potential in a QGP via the AdS/CFT correspondence 5

$\begin{array}{lll}2.1 .3 & \text { Shock waves in } \mathrm{AdS}_{5} & 6\end{array}$

2.1.4 The GYP dual conjecture 8

2.2 Remarks about the regularization of TS calculations in the case of wall-on$\begin{array}{ll}\text { wall collisions } & 11\end{array}$

3 Holographic QGP phase diagram for the central heavy-ion collisions $\quad 15$

4 Holographic QGP phase diagram in the wall-on-wall dual model of heavy$\begin{array}{lr}\text { ion collisions } & \mathbf{1 7}\end{array}$

4.1 Charged wall as a dual model for a heavy ion with a nonzero chemical potential 17

4.2 Charged wall-on-wall collision as a dual model for heavy-ion collisions with $\begin{array}{ll}\text { a nonzero chemical potential } & 18\end{array}$

$\begin{array}{ll}4.3 \text { Comparison of results } & 20\end{array}$

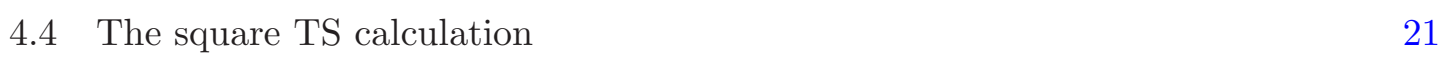

$\begin{array}{lll}4.5 & \text { Remarks about the regularization } & 23\end{array}$

5 Conclusion $\quad 25$

$\begin{array}{lll}5.1 \text { Summary } 25 & 25\end{array}$

$\begin{array}{lll}5.2 & \text { Further directions } & 25\end{array}$

A Metric of an ultrarelativistic charge in an AdS background 26

\section{Introduction}

For the last decade, since the publication of the fascinating papers [1-3], it has been realized that supersymmetric and nonsupersymmetric theories in the strong coupling limit could in principle be quite close in their properties [4]. The AdS/CFT correspondence, which appeared as a formal duality between the $\mathcal{N}=4$ super Yang-Mills theory and quantum gravity in the AdS background, has become a powerful tool for studying various properties of real physical systems in the strong-coupling limit [5]. An important branch of these investigations is the analysis of the quark-gluon plasma (QGP) from the standpoint of AdS 
holography (see, e.g., $[6,7]$ ). These applications of the AdS/CFT correspondence to the strongly coupled QGP have been mostly related to equilibrium properties of the plasma or to its kinetics/hydrodynamics near equilibrium.

A particular application of the AdS/CFT correspondence to the strongly coupled QGP is the analysis of the thermalization of matter and early entropy production immediately after the collision of relativistic heavy ions. The RHIC experiments have shown that a QGP forms at a very early stage just after the heavy-ion collision, i.e., a rapid thermalization occurs, and the QGP produced in the RHIC is believed to be strongly coupled, as is evidenced by its rapid equilibration. Strong collective flows and strong jet quenching are well reproduced by hydrodynamics [8-10]. This obviously requires calculating the strongly coupled field theory in a nonequilibrium process.

Gubser, Yarom, and Pufu recently proposed the gravitational shock wave in $\mathrm{AdS}_{5}$ as a possible holographic dual for the heavy ion and related the area of the trapped surface (TS) formed in a collision of such waves to the entropy of matter formed after the collision of heavy ions [13]. Early papers mentioning an analogy between colliding heavy ions and colliding gravitational shock waves in AdS space include [14]-[18]. This AdS-holographic model was also used to find the stress-energy tensor of the QGP formed by ion collision. According to the AdS/CFT dictionary, this stress-energy tensor is dual to the space-time metric after the shock-wave collision [18]. Analytic calculations that involve the analogy between colliding heavy ions and colliding gravitational shock waves have also been extensively studied recently [19]-[27].

The main result in $[13,28]$, confirmed by numerical calculations reported in $[29,30]$, is that in the limit of a very large collision energy $E$, the multiplicity (the entropy $S$ ) increases as

$$
S>\mathcal{C} E^{2 / 3}
$$

where $\mathcal{C}$ is a numerical factor (see section 2.1.4).

Alvarez-Gaume et al. considered the central collision of shock waves sourced by a nontrivial matter distribution in the transverse space and found a critical phenomenon occurring as the shock wave reaches some dilution limit [31]. This criticality may be related to the criticality found in [29], where the numerical results show the existence of a simple scaling relation between the critical impact parameter and the energy of colliding waves.

The size of colliding nuclei is introduced via the distance of those objects from the boundary along the holographic coordinate $z$.

Shuryak and Lin proposed a model of an infinite homogenous wall [29], which was analyzed in $[29,32]$. The advantage of this model is the essential simplicity of the calculations. But the legitimacy of these calculations requires some additional examination (see our discussion in section 2.2).

In heavy-ion collisions, the energy per nucleus is not the only important variable. Associating different nuclei with different kinds of shock waves can be tried. There are several proposals in the literature on this subject. For example, a holographic model with the UV part of the bulk geometry cut off was proposed in [33]. The formation of TSs in head-on collisions of charged shock waves in the (A)dS background was considered in [34], where it 
was shown that the formation of TSs on the past light cone is only possible when the charge is below a certain critical value, a situation similar to the collision of two ultrarelativistic charges in the Minkowski space-time [35]. This critical value depends on the energy of colliding particles and the value of a cosmological constant. The formation of TSs in head-on collisions of shock waves in gravitational theories with more complicated bulk dynamics, in particular, with the Einstein-dilaton dynamics, claimed to describe a holographic physics that is closer to QCD than the purely AdS theory [33, 36, 37], was recently considered by Kiritsis and Taliotis [38], ${ }^{1}$ who found that the multiplicity increases as

$$
S \gtrsim E^{0.24}
$$

which is rather close to the experimental data.

Here, we propose to incorporate the study of collisions of charged gravitational shock waves [34] into the description of colliding nuclei with a nonzero baryon chemical potential. In the holographic context, the chemical potential of a strongly coupled QGP on the gravity side is related to the temporal component $A_{t}$ of the $\mathrm{U}(1)$ gauge field [40]-[47]. The asymptotic value of this gauge field component in the bulk is interpreted as the chemical potential in the gauge theory

$$
\mu=\left.A_{t}\right|_{\text {boundary }}
$$

We use the same identification (1.3) for colliding ions. It would be interesting to calculate for the off-center collision of charged gravitational waves or generally smeared charged shock waves. Postponing this problem for further investigations, we here consider the head-on collision of charged point shock waves and charged wall shock waves. This provides the holographic picture for the QGP phase diagram in the first moment after collisions of heavy ions. These phase diagrams of the chemical potential (charge) $\mu$ versus the temperature (energy) $T$ are displayed in figures 5 and 11. The colored lines separate the TS phase from the phase with no TS. We note that the obtained diagrams differ from the phase diagram for the equilibrium QGP (see figure 1 in section 2.1.2).

This paper is organized as follows. In section 2, we set up the problem, describe the role of black holes (BHs) in the AdS/CFT description of a strongly coupled QGP in section 2.1.1, describe the QGP chemical potential in the AdS/CFT correspondence in section 2.1.2, recall the main facts about shock waves in $\mathrm{AdS}_{5}$ related to the TS formation in section 2.1.3, and describe the dual conjecture proposed in [13] in detail in section 2.1.4. In section 2.2 , we pay special attention to the regularization problem that appears in the wall shock wave approach. In section 3 , we present the phase diagram of chemical potential versus temperature for the QGP formed in the heavy-ion collisions using the holographic approach with the central collision of charged shock waves. In section 4 , we calculate the same problem using the regularized version of the charged wall shock waves. In section 5 , we summarize our calculations and also discuss further directions related to the holographic description of the QGP formed in heavy-ion collisions.

\footnotetext{
${ }^{1}$ The collision of dilatonic shock waves in the flat background was considered in [39].
} 


\section{Set up}

\subsection{Dual conjectures}

\subsubsection{Black holes and the AdS/CFT correspondence for a strongly coupled QGP}

The idea of using the AdS/CFT correspondence to describe the QGP is based on the possibility of establishing a one-to-one correspondence between phenomenological/thermodynamic plasma parameters $(T, E, P$, and $\mu)$ and the parameters characterizing $\mathrm{AdS}_{5}$ deformations. In the dual gravity setting, the source of temperature and entropy are attributed to the gravitational horizons. The relation between the energy density and temperature typical for the $\mathrm{BH}$ in the AdS according to $[48,49]$ is

$$
E=\frac{3 \pi^{3} L^{3}}{16 G_{5}} T^{4}
$$

In the phenomenological model of a QGP, such as the Landau or Bjorken hydrodynamic models $[50,51]$, the plasma is characterized by a space-time profile of the energy-momentum tensor $T_{\mu \nu}\left(x^{\rho}\right), \mu, \nu, \rho=0, \ldots, 3$. This state has its counterpart on the gravity side as a modification of the geometry of the original $\mathrm{AdS}_{5}$ metric. This follows the general AdS/CFT line: operators in the gauge theory correspond to fields in SUGRA. In the case of the energy-momentum tensor, the corresponding field is just the five-dimensional metric. It is convenient to parameterize the corresponding five-dimensional geometry as

$$
d s^{2}=L^{2} \frac{g_{\mu \nu}\left(x^{\rho}, z\right) d x^{\mu} d x^{\nu}+d z^{2}}{z^{2}},
$$

which is the five-dimensional Fefferman-Graham metric [52]. The flat case $g_{\mu \nu}=\eta_{\mu \nu}$ parameterizes $\mathrm{AdS}_{5}$ in Poincaré coordinates. The conformal boundary of the space-time is at $z=0$ and

$$
g_{\mu \nu}\left(x^{\rho}, z\right)=\eta_{\mu \nu}+z^{4} g_{\mu \nu}^{(4)}\left(x^{\rho}\right)+\ldots
$$

The AdS/CFT duality leads to the relation

$$
g_{\mu \nu}^{(4)}\left(x^{\rho}\right)=\frac{N_{\mathrm{c}}^{2}}{2 \pi^{2}}\left\langle T_{\mu \nu}\left(x^{\rho}\right)\right\rangle,
$$

where $N_{\mathrm{c}}$ is a number of colors (see [53] for a brief review).

Applying the AdS/CFT correspondence to the hydrodynamic description of the QGP is based on the fact that the energy-momentum tensor can be obtained directly from the expansion of the $\mathrm{BH}$ in $\mathrm{AdS}_{5}$ metric (2.3) corresponding to the simple hydrodynamic model

$$
\left\langle T_{\mu \nu}\right\rangle \propto g_{\mu \nu}^{(4)}=\operatorname{diag}\left(\frac{3}{z_{0}^{4}}, \frac{1}{z_{0}^{4}}, \frac{1}{z_{0}^{4}}, \frac{1}{z_{0}^{4}}\right) .
$$

The $\mathrm{AdS}_{5} \mathrm{BH}$ in the Fefferman-Graham coordinates has form (2.2) with the following nonzero components of $g_{\mu \nu}\left(x^{\rho}, z\right)$ (see $[6,7]$ and the references therein):

$$
g_{00}=-\frac{\left(1-z^{4} / z_{0}^{4}\right)^{2}}{1+z^{4} / z_{0}^{4}}, \quad g_{i i}=\left(1+\frac{z^{4}}{z_{0}^{4}}\right) .
$$


The change of coordinates $\tilde{z}=z /\left(1+z^{4} / z_{0}^{4}\right)^{1 / 2}$ transforms (2.2) into the standard metric form of the AdS-Schwarzschild static BH

$$
\tilde{z}^{2} d s^{2}=-\left(1-\frac{\tilde{z}^{4}}{\tilde{z}_{0}^{4}}\right) d t^{2}+d \vec{x}^{2}+\frac{1}{1-\tilde{z}^{4} / \tilde{z}_{0}^{4}} d \tilde{z}^{2},
$$

where $\tilde{z}_{0}=z_{0} / \sqrt{2}$ is the horizon location.

\subsubsection{The chemical potential in a QGP via the AdS/CFT correspondence}

The Reissner-Nordström (RN) metric in the AdS space has the form

$$
\begin{gathered}
d s^{2}=-g(R) d T^{2}+g(R)^{-1} d R^{2}+R^{2} d \Omega_{D-2}^{2}, \\
g(R)=1-\frac{2 M}{R^{2}}+\frac{Q^{2}}{R^{4}}+\frac{\Lambda}{3} R^{2},
\end{gathered}
$$

where $\Lambda$ is a cosmological constant, $\Lambda / 3 \equiv 1 / a^{2}, M$ and $Q$ are related to the ArnowittDeser-Misner mass $m$ and the charge $\sigma$,

$$
M=\frac{4 \pi G_{5} m}{3 \pi^{2}}, \quad Q^{2}=\frac{4 \pi G_{5} \sigma^{2}}{3} .
$$

and $\sigma$ is the charge of the electromagnetic field (purely electric) with only one nonzero component

$$
A=A_{T} d T=\left(-\sqrt{\frac{3}{4}} \frac{Q}{R^{2}}+\Phi\right) d T
$$

where $\Phi$ is the constant

$$
\Phi=\frac{\sqrt{3}}{2} \frac{Q}{R_{+}^{2}}
$$

and $R_{+}$is the largest real root of $g(R)$. The thermodynamics of the charged $\mathrm{BH}$ is described by the grand canonical potential (free energy) $W=I / \beta$, the Hawking temperature $T=$ $1 / \beta$, and the chemical potential [54-57] given by

$$
I=\frac{\pi \beta}{8 G_{5} a^{2}}\left(a^{2} R_{+}^{2}+R_{+}^{4}-\frac{Q^{2} a^{2}}{R_{+}^{2}}\right), \quad T=\frac{1}{4 \pi} g^{\prime}\left(R_{+}\right), \quad \mu=\frac{\sqrt{3} Q}{2 R_{+}^{2}},
$$

where $R_{+}$is the outer horizon, $g\left(R_{+}\right)=0$, and $I$ is given by the value of the action at $(2.9)$ and (2.11). The relation to the first law of thermodynamics, $d \mathcal{E}=T d \mathcal{S}+\mu d \mathcal{Q}$, is obtained under the identifications

$$
W=\mathcal{E}-T \mathcal{S}-\Phi \mathcal{Q}, \quad \mathcal{E}=m, \quad \mathcal{S}=\frac{S_{H}}{4 G_{5}}, \quad \mathcal{Q}=q, \quad \mu=\Phi
$$

We note that just the asymptotic value of a single gauge field component gives the chemical potential [40]-[47]

$$
\mu=\lim _{r \rightarrow \infty} A_{t}(r)
$$

The QGP is characterized at least by two parameters: the temperature and the chemical potential. Generally speaking, quantum field theories can have nonzero chemical potentials for any or all of their Noether charges. In the AdS/CFT context, two different types of chemical potential are considered: related to the R-charge and to the baryon number. 


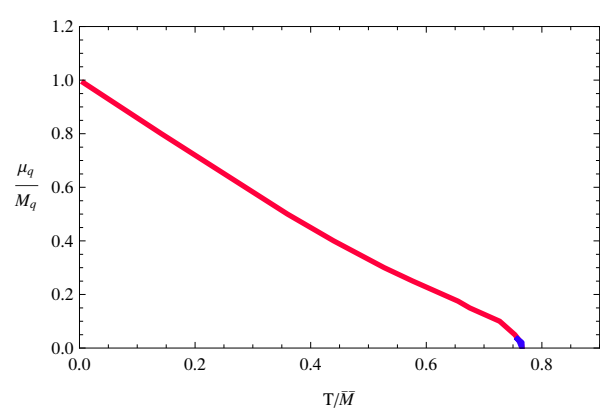

(a)

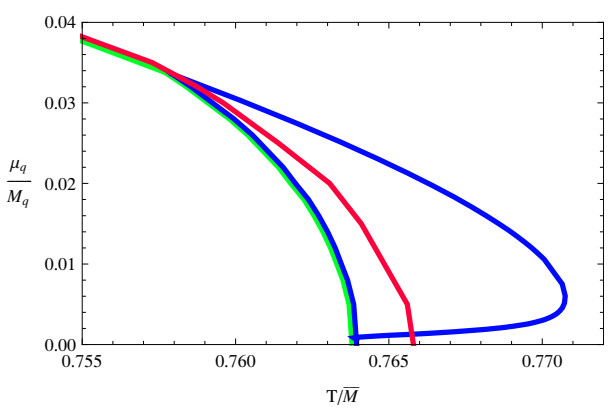

(b)

Figure 1. Phase diagram from [40]: (a) The red line in the quark chemical potential $\mu_{q} / M_{q}$ versus the temperature $T / \bar{M}$ phase space separates the phase of Minkowski embeddings (small temperatures, small $\mu_{q} / M_{q}$ ) from the phase of $\mathrm{BH}$ embeddings (see [40] for the details). (b) An enlargement of the region near the end of the line in (a): different lines in correspond to different embedding geometries.

The baryon number charge can only occur when we have a theory containing fundamental flavors. Introducing flavors into the gauge theory via a D7 brane leads to the appearance of a $\mathrm{U}\left(N_{\mathrm{f}}\right)$ global flavor symmetry. The flavor group contains $\mathrm{U}(1)_{\mathrm{B}}$, i.e., a baryon number symmetry, and a chemical potential $\mu_{\mathrm{B}}$ is added for this baryon number [41]. To calculate the free energy, we must calculate the Dirac-Born-Infeld action for a D7 brane. We note that there is a divergence in the formal definition, and we must hence go through the renormalization process (see, e.g., the lectures in [58] and also see [59]).

The R-charge chemical potential appears for SUSY models [47]. In the $N=1$ case, there is a U(1) R-symmetry group. In the extended SUSY case, for example, in the $N=2$ case, the quark mass term breaks the R-symmetry.

A typical phase diagram of chemical potential versus temperature is presented in figure 1 (the diagram is taken from [40]). In the phase diagram, $\mu_{q}=\mu_{\mathrm{B}} / N_{\mathrm{c}}, \mu_{q}$ is the quark chemical potential, $\bar{M} \propto m_{q}$ is a mass scale defined as $\bar{M}=2 M_{q} / \sqrt{\lambda}$, and $\lambda=g_{Y M}^{2} N_{\mathrm{c}}$.

\subsubsection{Shock waves in $\mathrm{AdS}_{5}$}

Shock waves propagating in the AdS space have the form

$$
d s^{2}=L^{2} \frac{-d u d v+d x_{\perp}^{2}+\phi\left(x_{\perp}, z\right) \delta(u) d u^{2}+d z^{2}}{z^{2}},
$$

where $u$ and $v$ are light-cone coordinates and $x_{\perp}$ is the coordinate transverse to the direction of motion of the shock wave and to the $z$ direction. This metric is sourced by the stressenergy-momentum tensor $T_{M N}$ with only one nonzero component $T_{u u}^{\mathrm{SW}}$,

$$
T_{u u}^{\mathrm{SW}}=J_{u u}\left(z, x_{\perp}\right) \delta(u) .
$$

and the Einstein equation of motion reduces to

$$
\left(\square_{H_{3}}-\frac{3}{L^{2}}\right) \Phi\left(z, x_{\perp}\right)=-16 \pi G_{5} \frac{z}{L} J_{u u}\left(z, x_{\perp}\right),
$$


where

$$
\Phi\left(z, x_{\perp}\right) \equiv \frac{L}{z} \phi\left(z, x_{\perp}\right)
$$

and

$$
\square_{H_{3}}=\frac{z^{3}}{L^{2}} \frac{\partial}{\partial z} z^{-1} \frac{\partial}{\partial z}+\frac{z^{2}}{L^{2}}\left(\frac{\partial^{2}}{\partial x_{\perp}^{2}}\right) .
$$

Different forms of the shock waves correspond to different forms of the source $J_{u u}\left(z, x_{\perp}\right)$. The most general $O(3)$-invariant shock wave in the AdS space located at $u=0$ corresponds to

$$
\Phi^{O(3)}\left(z, x_{\perp}\right)=F(q),
$$

where $q$ is the chordal distance

$$
q=\frac{\left(x_{\perp}^{1}\right)^{2}+\left(x_{\perp}^{2}\right)^{2}+\left(z-z_{0}\right)^{2}}{4 z z_{0}},
$$

In this case $\rho$, related to $J_{u u}$ as

$$
\frac{z}{L} J_{u u}\left(z, x_{\perp}\right) \equiv \rho\left(z, x_{\perp}\right),
$$

has the form

$$
\rho^{O(3)}\left(z, x_{\perp}\right)=\rho(q),
$$

and the Einstein equation of motion becomes

$$
\left(\square_{H_{3}}-\frac{3}{L^{2}}\right) F=-16 \pi G_{5} \rho(q)
$$

or, explicitly,

$$
q(q+1) F_{q q}^{\prime \prime}+(3 / 2)(1+2 q) F_{q}^{\prime}-3 F=-16 \pi G_{5} L^{2} \rho(q) .
$$

The point shock wave shape $F^{\mathrm{p}}$ is given by the solution of (2.17) with

$$
J_{u u}=E \delta(u) \delta(z-L) \delta\left(x^{1}\right) \delta\left(x^{2}\right)
$$

and has the form

$$
F^{\mathrm{p}}\left(z, x_{\perp}\right)=\frac{8 L^{2} G_{5} E z^{3}}{\left(x_{\perp}^{2}+(z-L)^{2}\right)^{3}} .
$$

This point shock wave shape is in fact equal to $F^{\mathrm{P}}(q), \Phi^{\mathrm{P}}\left(z, x_{\perp}\right)=F^{\mathrm{P}}(q)$, which is a solution of $(2.25)$ with

$$
\rho^{\mathrm{p}}(q)=\frac{E}{L^{3}} \frac{\delta(q)}{\sqrt{q(1+q)}} .
$$

It has the form

$$
F^{\mathrm{p}}=\frac{2 G_{5} E}{L}\left(\frac{\left(8 q^{2}+8 q+1\right)-4(2 q+1) \sqrt{q(1+q)}}{\sqrt{q(1+q)}}\right) .
$$

The shape of the charged point shock wave is a sum of two components (see appendix A for the calculation details)

$$
F=F^{\mathrm{p}}+F^{Q},
$$


where $F^{\mathrm{p}}$ is given by $(2.29)$ and $F^{Q}$ is the solution of (2.25) with

$$
\rho^{\mathrm{p} Q}=\frac{5 \bar{Q}^{2}}{32 \cdot 64 L^{5} G_{5}} \frac{1}{[q(q+1)]^{5 / 2}}=\frac{5 Q_{n}^{2}}{\pi 24 \cdot 64 L^{5}} \frac{1}{[q(q+1)]^{5 / 2}}
$$

or, explicitly,

$$
F^{Q}=\frac{5 G_{5} Q_{n}^{2}}{48 L^{3}} \frac{144 q^{2}+16 q-1+128 q^{4}+256 q^{3}-64(2 q+1) q(q+1) \sqrt{q(1+q)}}{q(1+q) \sqrt{q(1+q)}} .
$$

To establish the connection with [34], we note the notational relations

$$
\begin{aligned}
\bar{M} & =\frac{4 G_{5} E}{3 \pi} \\
\bar{Q}^{2} & =\frac{4 G_{5} Q_{n}^{2}}{3 \pi},
\end{aligned}
$$

and

$$
\begin{aligned}
& \left.\frac{3 \pi \bar{M}}{2 a}\right|_{\text {notation in [34] }}=\left.\frac{2 G_{5} E}{L}\right|_{\text {notation in [13] and here }}, \\
& \left.\frac{5 \pi \bar{Q}^{2}}{64 a^{3}}\right|_{\text {notation in [34] }}=\left.\frac{5 G_{5} Q_{n}^{2}}{48 L^{3}}\right|_{\text {notation here }} \cdot
\end{aligned}
$$

More complicated shock waves in AdS and dS spaces were considered in [60-65].

\subsubsection{The GYP dual conjecture}

Gubser, Yarom, and Pufu (GYP) proposed the following holographic picture for colliding nuclei dual to QCD [13]:

- the bulk dual of the boundary nuclei is the shock waves of form (2.15) propagating in the AdS space;

- the bulk dual of two colliding nuclei in the bulk is the line element for two identical shock waves propagating towards one another in the AdS space,

$$
d s^{2}=L^{2} \frac{-d u d v+d x_{\perp}^{2}+\phi_{1}\left(x_{\perp}, z\right) \delta(u) d u^{2}+\phi_{2}\left(x_{\perp}, z\right) \delta(v) d v^{2}+d z^{2}}{z^{2}} ;
$$

- when the shock waves collide in the bulk, a BH should form, signifying the formation of a QGP.

The TS technic $[66,67]$ is usually used to estimate the $\mathrm{BH}$ formation. $^{2}$ A TS is a surface whose null normals all propagate inward [69, 70]. There is no rigorous proof that the TS formation in the asymptotically AdS space-time provides the $\mathrm{BH}$ formation, but there is a common belief that TSs must lie behind an event horizon and that a lower bound on the entropy $S_{\mathrm{AdS}}$ of the $\mathrm{BH}$ is given by the TS area $A_{\text {trapped }}$,

$$
S_{\text {AdS }} \geq S_{\text {trapped }} \equiv \frac{A_{\text {trapped }}}{4 G_{5}} .
$$

\footnotetext{
${ }^{2}$ This estimate can be also obtained using the so-called capture arguments $[39,68]$.
} 
The relations between the bulk parameters $G_{5}, L$, and $E$ and the QGP phenomenological parameters must be fixed to make the proposed duality prescription more precise. According to [33], one of these relations is

$$
\frac{L^{3}}{G_{5}}=\frac{16 E \cdot T^{4}}{3 \pi^{3}}=\frac{11 \cdot 16}{3 \pi^{3}} \approx 1.9
$$

The arguments supporting (2.39) are as follows. Lattice calculations for the QGP in [71] showed that $E T^{4}$ is a slowly varying quantity and

$$
\frac{E}{T^{4}} \approx 11
$$

Just to match BH equation of state (2.1) to (2.40), GYP assumed (2.39) (see [13]). It is important that an identification of the total energy of each nucleus with the energy of the corresponding shock wave is assumed here. We can modify this identification and assume that only a part of the gravitational shock wave energy is related to the total energy of the nucleus.

The AdS/CFT dual relation (2.4) between the expectation value of the gauge theory stress tensor and the $\mathrm{AdS}_{5}$ metric deformation by the shock wave was used in [13] to fix the dimensionless parameter $E L$ :

$$
\left\langle T_{u u}\right\rangle=\frac{L^{2}}{4 \pi G_{5}} \lim _{z \rightarrow 0} \frac{1}{z^{3}} \Phi\left(z, x_{\perp}\right) \delta(u) .
$$

For the point shock wave $\Phi^{\mathrm{p}}$ given by $(2.27)$, we obtain the stress tensor in the boundary field theory

$$
\left\langle T_{u u}\right\rangle=\frac{2 L^{4} E}{\pi\left(L^{2}+\left(x^{1}\right)^{2}+\left(x^{2}\right)^{2}\right)^{3}} \delta(u) .
$$

The right-hand side of (2.42) depends on the total energy $E$ and $L$, and $L$ has the meaning of the root-mean-square radius of the transverse energy distribution. It was assumed in [13] that $L$ is equal to the root-mean-square transverse radius of the nucleons, which in accordance with a Woods-Saxon profile for the nuclear density [72, 73] is of the order of a few fm. In particular, it is equal to $L \approx 4.3 \mathrm{fm}$ for $\mathrm{Au}$ and $L \approx 4.4 \mathrm{fm}$ for $\mathrm{Pb}$. However in principle $\mathrm{L}$ here is an arbitrary parameter, that can be fixed to fit the experimental data.

The RHIC collides Au nuclei $(\mathrm{A}=197)$ at $\sqrt{s_{N N}}=200 \mathrm{GeV}$. This means that each nucleus has the energy $E=100 \mathrm{GeV}$ per nucleon, for a total of about $E=E_{\text {beam }}=$ 19.7 TeV for each nucleus.

The LHC will collide $\mathrm{Pb}$ nuclei $(\mathrm{A}=208)$ at $\sqrt{s_{N N}}=5.5 \mathrm{TeV}$, which means $E=$ $E_{\text {beam }}=570 \mathrm{TeV}$.

The estimates in [13] for the dimensionless values $E L$ for $\mathrm{Au}-\mathrm{Au}$ and $\mathrm{Pb}-\mathrm{Pb}$ collisions are

$$
\begin{gathered}
\left.E L\right|_{\mathrm{Au}-\mathrm{Au}, \sqrt{s_{N N}}=200 \mathrm{GeV}} \approx 4.3 \times 10^{5}, \\
\left.E L\right|_{\mathrm{Pb}-\mathrm{Pb}, \sqrt{s_{N N}}=5.5 \mathrm{TeV}} \approx 1.27 \times 10^{7} .
\end{gathered}
$$


We note that tuning the scale $L$ or $z_{0}$ of the bulk colliding object to the size of the nucleus or to the "saturation scale" $Q_{s}$ in the "color glass" models was proposed in [29].

The calculations in [13] show that in the limit of a very large collision energy $E$, the entropy increases as $E^{2 / 3}$,

$$
S_{\text {trapped }} \approx \pi\left(\frac{L^{3}}{G_{5}}\right)^{1 / 3}(2 E L)^{2 / 3} .
$$

Considering off-center collisions of gravitational shock waves in the AdS space do not change the scaling $E^{2 / 3}$. But a critical impact parameter, beyond which the TS does not exist, was observed in [29] (cf. the result in [31]). Experimental indications for a similar critical impact parameter in real collisions had been noted [29].

The relation of the total multiplicity $S_{\mathrm{QGP}}$ (given by experimental data) to the entropy $S_{\text {AdS }}$ produced in the gravitational wave collision in $\mathrm{AdS}_{5}$ has some subtleties [33]. Phenomenological considerations $[13,74,75]$ lead to estimating the total multiplicity $S_{\mathrm{QGP}}$ by the number $N_{\mathrm{ch}}$ of charged particles times a factor $\sim 7.5$,

$$
S_{\mathrm{QGP}} \approx 7.5 N_{\mathrm{ch}}
$$

The TS analysis does not give the produced entropy, but it provides a lower bound,

$$
S_{\text {trapped }} \leq S_{\text {AdS }}
$$

Taking into account that in the calculations in [13], the gravitational shock wave energy was identified with the energy of colliding ions and $L$ was identified with the nucleus size, we can introduce proportionality constants between these quantities and obtain

$$
\mathcal{M} \cdot S_{\text {trapped }}<N_{\mathrm{ch}}
$$

where all proportionality factors are included in the overall factor $\mathcal{M}$. We can take $\mathcal{M}$ to fit the experimental data at some point. But the scaling $S_{\text {trapped }} \propto s_{N N}^{1 / 3}$ implied by (2.45) differs from the observed scaling, which is closer to the dependence $S \propto s_{N N}^{1 / 4}$, which is predicted by the Landau model [50] (see figure 2). Obviously, we can avoid a conflict between [13] and the experiment if $E<E_{\max }$, but if $\mathrm{E}$ can be arbitrary large, then the conflict arises.

In figure 2, we plot the dependence of entropy bound (2.45) on the energy together with the curve schematically representing the realistic curve that fits the experimental data [76]. It can be seen that by changing the coefficient $\mathcal{M}$, we can avoid the conflict only for energy up to some $E_{\max }$. We chose the overall coefficient of the numerical plot to fit the RHIC data [76], which are indicated by dots in figure 2 .

In the above estimate, the energy of each shock wave is identified with the energy of colliding beams. As was noted in [28], the fit to the data can be improved by identifying the energy of each shock wave with the fraction of the energy of the nucleus carried by a nucleus participating in the collision. This gives an extra parameter for fitting the data. But a conflict still arises at high energies. It was proposed in [28] to solve this problem by removing a UV part of the AdS bulk. Shock waves corresponding to the $\mathrm{BH}$ with a nonzero dilaton field $[36,37]$ were considered in [38], where it was shown that the lower bound on $N_{\mathrm{ch}}$ scales is closer to $s_{N N}^{1 / 4}$. 


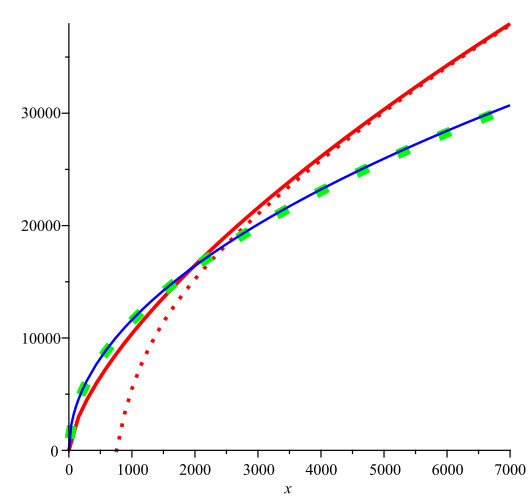

(a)

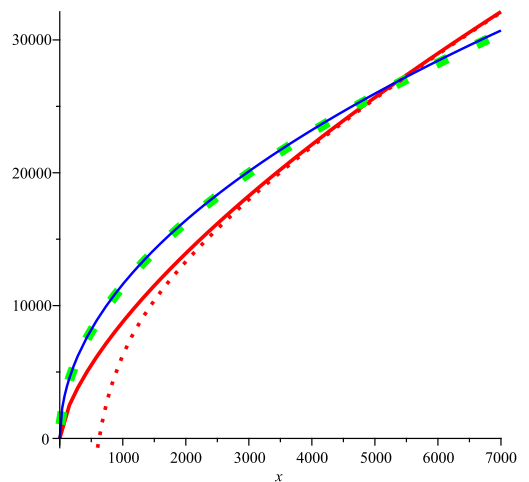

(b)

Figure 2. (color on-line) Plots of the total number of charged particles versus energy: the red lines represent estimate (2.45). The plots in (a) and (b) differ by the overall factor $\mathcal{M}$. The blue lines correspond to the prediction of the Landau model, and the dotted green lines schematically represent the curves that fit the experimental data. The dashed lines correspond to corrections to the GYP multiplicity via a nonzero chemical potential (see section 3).

\subsection{Remarks about the regularization of TS calculations in the case of wall- on-wall collisions}

A much simpler dual description of the colliding nuclei using a wall-on-wall collision in the bulk was proposed in [29]. The Einstein equation for the profile of the wall shock wave [29] has the form

$$
\left(\partial_{z}^{2}-\frac{3}{z} \partial_{z}\right) \phi(z)=J_{u u}^{\mathrm{WP}}, \quad J_{u u}^{\mathrm{WP}}=-16 \pi G_{5} \frac{E}{L^{2}} \frac{z_{0}^{3}}{L^{3}} \delta\left(z-z_{0}\right)
$$

To find a TS that can be formed in the collision of two wall shock waves, we must find a solution of Einstein equation (2.49) satisfying two conditions. It is convenient to write these conditions in terms of the function $\psi(z)$ related to $\phi$ by

$$
\phi(z)=\frac{z}{L} \psi
$$

They have the forms

$$
\begin{aligned}
& \psi\left(z_{a}\right)=\psi\left(z_{b}\right)=0, \\
& \psi^{\prime}\left(z_{a}\right) \frac{z_{a}}{L}=2, \quad \psi^{\prime}\left(z_{b}\right) \frac{z_{b}}{L}=-2,
\end{aligned}
$$

where $z_{a}$ and $z_{b}$ are assumed to be the boundaries of the TS [29]. But as is seen below, strictly speaking, we cannot call the solution of (2.49) with boundary conditions (2.51) and (2.52) the TS, because this surface is assumed to be smooth and compact by definition, while the solution in [29] is nonsmooth and noncompact. We therefore call the solution found in [29] a quasi-TS.

We recall the construction presented in [29], where the solution of Einstein equation (2.49) was written such that property (2.51) is automatically satisfied. This solution 
has the form

$$
\psi(z)=\psi_{a}(z) \Theta\left(z_{0}-z\right)+\psi_{b}(z) \Theta\left(z-z_{0}\right),
$$

where

$$
\begin{aligned}
& \psi_{a}(z)=-\frac{4 G \pi E\left(z_{0}^{4} / z_{b}^{4}-1\right) z_{b}^{4} z_{a}^{3}\left(z^{3} / z_{a}^{3}-z_{a} / z\right)}{L^{4}\left(z_{b}^{4}-z_{a}^{4}\right)}, \\
& \psi_{b}(z)=-\frac{4 G \pi E\left(z_{0}^{4} / z_{a}^{4}-1\right) z_{a}^{4} z_{b}^{3}\left(z^{3} / z_{b}^{3}-z_{b} / z\right)}{L^{4}\left(z_{b}^{4}-z_{a}^{4}\right)} .
\end{aligned}
$$

We first note that solution (2.53) is nonsmooth and can be decomposed as

$$
\psi_{a}=\Xi^{(\mathrm{sm})}+\Xi^{\mathrm{nonsm}} .
$$

The nonsmooth part of it is

$$
\begin{aligned}
\Xi^{\text {nonsm }} & =\frac{\mathcal{K}}{z}\left(-\frac{z_{b}}{z_{a}^{3}} \Upsilon_{1}-\frac{z_{a}}{z_{b}^{3}} \Upsilon_{2}\right), \\
\Upsilon_{1} & =z^{4} \Theta\left(z_{0}-z\right)+z_{0}^{4} \Theta\left(z-z_{0}\right), \\
\Upsilon_{2} & =z_{0}^{4} \Theta\left(z_{0}-z\right)+z^{4} \Theta\left(z-z_{0}\right),
\end{aligned}
$$

where

$$
\mathcal{K}=\frac{4 G \pi E}{L^{4}} \frac{z_{a}^{3} z_{b}^{3}}{z_{b}^{4}-z_{a}^{4}}
$$

To smooth the solution, we must therefore smooth the function $\Xi$. We can do this by regularizing the Heaviside step function

$$
\begin{aligned}
& \Theta\left(z_{0}-z\right) \approx \Gamma_{1}=\frac{\arctan \left(R^{3}\left(z_{0}-z\right)^{3}\right)}{\pi}+\frac{1}{2}, \\
& \Theta\left(z-z_{0}\right) \approx \Gamma_{2}=\frac{\arctan \left(R^{3}\left(z-z_{0}\right)^{3}\right)}{\pi}+\frac{1}{2}
\end{aligned}
$$

and considering the regularized functions $\tilde{\Upsilon}_{1}$ and $\tilde{\Upsilon}_{2}$,

$$
\begin{aligned}
& \tilde{\Upsilon}_{1}=z^{4}\left(\frac{\arctan \left(R\left(z_{0}-z\right)\right)^{3}}{\pi}+\frac{1}{2}\right)+z_{0}^{4}\left(\frac{\arctan \left(R\left(z-z_{0}\right)\right)^{3}}{\pi}+\frac{1}{2}\right), \\
& \tilde{\Upsilon}_{2}=z_{0}^{4}\left(\frac{\arctan \left(R\left(z_{0}-z\right)\right)^{3}}{\pi}+\frac{1}{2}\right)+z^{4}\left(\frac{\arctan \left(R\left(z-z_{0}\right)\right)^{3}}{\pi}+\frac{1}{2}\right) .
\end{aligned}
$$

For derivatives, we have

$$
\begin{array}{ll}
\frac{d \Upsilon_{1}}{d z} \approx 4 z^{3} \Theta\left(z_{0}-z\right), & \left.\frac{d \tilde{\Upsilon}_{1}}{d z}\right|_{\text {approx }} \approx \frac{4 z^{3}\left(\arctan \left(R\left(z_{0}-z\right)\right)^{3}+\pi\right)}{\pi}, \\
\frac{d \Upsilon_{2}}{d z} \approx 4 z^{3} \Theta\left(z-z_{0}\right), & \left.\frac{d \tilde{\Upsilon}_{2}}{d z}\right|_{\text {approx }} \approx \frac{4 z^{3}\left(\arctan \left(R\left(z-z_{0}\right)\right)^{3}+\pi\right)}{\pi} .
\end{array}
$$




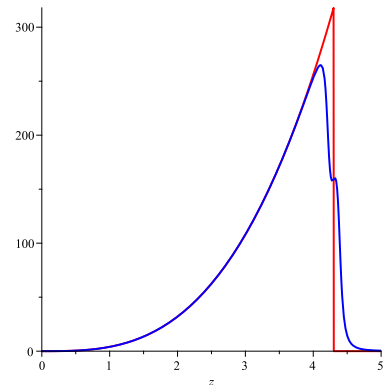

(a)

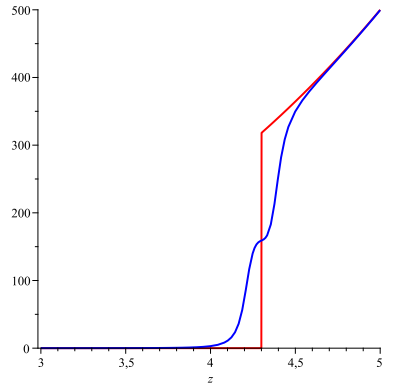

(b)

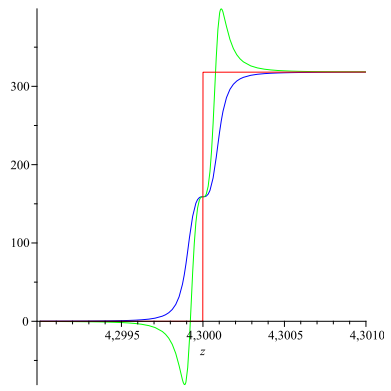

(c)

Figure 3. (a) The functions $d \Upsilon_{1} / d z$ (red line) and $d \tilde{\Upsilon}_{1} /\left.d z\right|_{\text {approx }}$ (blue line). (b) The functions $d \Upsilon_{2} / d z$ (red line) and $d \tilde{\Upsilon}_{2} /\left.d z\right|_{\text {approx }}$ (blue line). (c) The functions $d \Upsilon_{2} / d z$ (red line), $d \tilde{\Upsilon}_{2} /\left.d z\right|_{\text {approx }}$ (blue line), and $d \tilde{\Upsilon}_{2} / d z$ (green line). The regularization parameter is $R=10$ in (a) and (b) and $R=10^{4}$ in (c).

We present the derivatives of the functions $\Upsilon_{1}$ and $\Upsilon_{2}$ and also of the smoothed functions $\tilde{\Upsilon}_{1}$ and $\tilde{\Upsilon}_{2}$ in figure 3. For $R=10^{4}$ (see below), the differences between the derivatives $d \tilde{\Upsilon}_{i} / d z$ and their approximations given by (2.63) and (2.64),

$$
\begin{aligned}
& \Delta_{1}(z)=\frac{d \tilde{\Upsilon}_{1}}{d z}-\left.\frac{d \tilde{\Upsilon}_{1}}{d z}\right|_{\text {approx }}, \quad \Delta_{2}(z)=\frac{d \tilde{\Upsilon}_{2}}{d z}-\left.\frac{d \tilde{\Upsilon}_{2}}{d z}\right|_{\text {approx }}, \\
& \Delta_{1}(z)=-\Delta_{2}(z)=-3 \frac{z^{4} R^{3}\left(z_{0}-z\right)^{2}}{\left(1+R^{6}\left(z_{0}-z\right)^{6}\right) \pi}+3 \frac{z_{0}^{4} R^{3}\left(z-z_{0}\right)^{2}}{\left(1+R^{6}\left(z-z_{0}\right)^{6}\right) \pi},
\end{aligned}
$$

are of the order $\gtrsim 10^{-3} \mathrm{fm}^{3}$ only in the interval $z \in\left[z_{0}^{\prime}, z_{0}^{\prime \prime}\right]$, where $z_{0}^{\prime}=4.293 \mathrm{fm}$ and $z_{0}^{\prime \prime}=4.307 \mathrm{fm}$. Indeed, in our consideration (spread case), the largest value of $z_{a}$ is $4.260706906 \mathrm{fm}$, and the smallest value of $z_{b}$ is $4.340400579 \mathrm{fm}$. At the points $z_{0}^{\prime}=4.260706906 \mathrm{fm}$ and $z_{0}^{\prime \prime}=4.340400579 \mathrm{fm}$, the quantity $\Delta_{1}$ is less then $5 \cdot 10^{-6} \mathrm{fm}^{3}$. At the points $z_{0}^{\prime}=0.6948439783 \mathrm{fm}$ and $z_{0}^{\prime \prime}=1018.393720 \mathrm{fm}$, the quantity $\Delta_{1}$ is less then $2 \cdot 10^{-12} \mathrm{fm}^{3}$.

We present a schematic picture of the root locations and the region where $\left|\Delta_{i}(z)\right| \gtrsim$ $10^{-3}$ in figure 4 . It can be seen that the difference $\Delta_{i}$ is inessential in the root locations and that using approximations (2.63) and (2.64) is therefore acceptable. The regularized version of the function $\psi$ is

$$
\psi_{\text {reg }}=\psi_{a}(z) \Gamma_{1}+\psi_{b}(z) \Gamma_{2} .
$$

We must now impose conditions (2.52) on the regularized functions,

$$
\begin{aligned}
& \left.\frac{z_{a}}{2 L} \frac{d}{d z} \psi_{\mathrm{reg}}\right|_{z=\tilde{z}_{a}}=1, \\
& \left.\frac{z_{b}}{2 L} \frac{d}{d z} \psi_{\mathrm{reg}}\right|_{z=\tilde{z}_{b}}=-1,
\end{aligned}
$$

and find $\tilde{z}_{a}$ and $\tilde{z}_{a}$ from these conditions. But these calculations are difficult. Instead of finding $\tilde{z}_{a}$ from condition (2.68), we propose using a regularization that does not change $z_{a}$ 


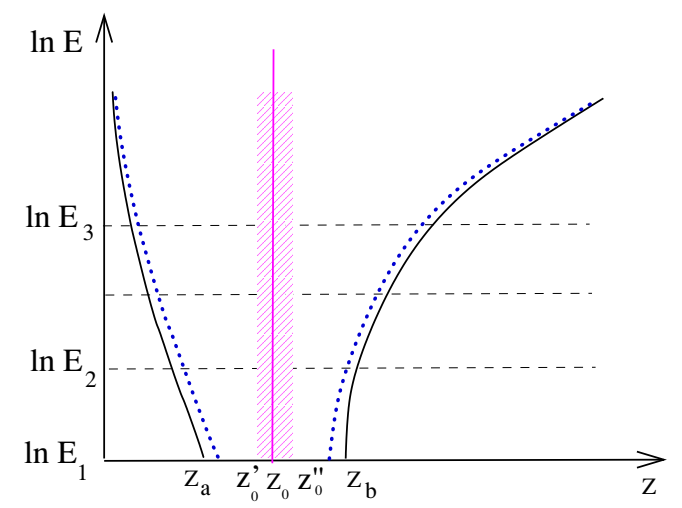

Figure 4. (color on-line) The schematic plot of root locations (solid black lines) depending on the energy (in the logarithmic scale) and the location of the differences $\left|d \tilde{\Upsilon}_{i} / d z-d \tilde{\Upsilon}_{i} / d z\right|_{\text {approx }} \mid \gtrsim 10^{-3}$, $i=1,2$ (the magenta-shaded region). The solid magenta line shows the location of the wall. The dotted blue lines show the location of zeros for the charged wall.

found from formal conditions (2.52). We can verify that the formal $z_{a}$ in fact also satisfies the regularized condition if the regularization is sufficiently smooth. We therefore take $z_{a}$ and substitute it in the left-hand side of regularized condition (2.68). We define

$$
\begin{aligned}
& \left.F_{a, \mathrm{reg}}\right|_{z=z_{a}}=\left.\frac{z_{a}}{2 L}\left(\frac{d \psi_{a}}{d z} \Gamma_{1}+\frac{d \psi_{b}}{d z} \Gamma_{2}\right)\right|_{z=z_{a}}=1+\delta_{1}, \\
& \left.F_{b, \mathrm{reg}}\right|_{z=z_{b}}=\left.\frac{z_{b}}{2 L}\left(\frac{d \psi_{a}}{d z} \Gamma_{1}+\frac{d \psi_{b}}{d z} \Gamma_{2}\right)\right|_{z=z_{b}}=-1+\delta_{2} .
\end{aligned}
$$

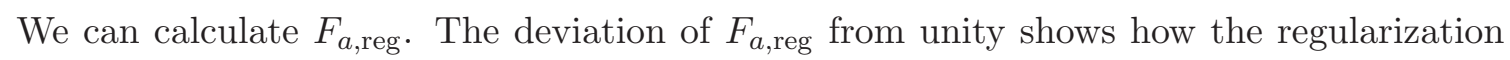
changes conditions (2.52). In the following table, we present the results of calculating $F_{a \text {,reg }}$ for a wide range of the theory parameter. We choose the parameter $R$ as minimally needed to make $\delta_{1}$ and $\delta_{2}$ negligible at energies in the range $10^{-4}<E<10^{2} \mathrm{TeV}$. Using direct numerical calculations, we choose $R=10^{4}$. We perform numerical calculations at $R=10^{4}$ and obtain the following table:

\begin{tabular}{|c|c|c|c|c|c|}
\hline$E, \mathrm{TeV}$ & $Q, \mathrm{fm}^{1 / 2}$ & $z_{a}, \mathrm{fm}$ & $z_{b}, \mathrm{fm}$, & $F_{a}$ & $F_{b}$ \\
\hline 118.2 & 0 & 0.04399350434 & $4.015208900 \cdot 10^{6}$ & 1.00000 & -1.00000 \\
30 & 0 & 0.06948439782 & $1.019088495 \cdot 10^{6}$ & 1.00000 & -1.00000 \\
0.03 & 0 & 0.6948439783 & 1018.393720 & 1.00000 & -1.00000 \\
0.00025 & 0 & 4.260706906 & 4.340400579 & 0.99999 & -0.99999 \\
\hline
\end{tabular}

It follows from the table that obviously $F_{a} \approx 1$ and $F_{b} \approx-1$.

As above, strictly speaking, we cannot regard an infinite surface as a TS of any kind. Nevertheless, we can assume that the transverse size of colliding objects is finite but very large, and boundary conditions therefore do not affect the gravitational interaction processes of the inner parts of sources. If we are interested only in the specific area of the 
formed TS with respect to the unit of shock wave area, then we can define it as

$$
\mathcal{A} \approx \lim _{L \rightarrow \infty} \frac{A_{\text {trap }}(L)}{A_{\text {source }}(L)}
$$

and the approximate equality holds because the boundary effects are negligible. As often happens, we can obtain answers for finite physical systems by calculating for infinite nonphysical objects.

\section{Holographic QGP phase diagram for the central heavy-ion collisions}

In this section, we construct the phase diagram for a TS formed in the central collision of two identical pointlike charged shock waves [34]. The profile of pointlike charged shock waves in the AdS space is given by (2.30) with (2.29) and (2.32). The existence of the TS in the central collision of two pointlike charged shock waves means the existence of a real solution $q_{0}$ of the equation (see [34] for the details)

$$
F^{\prime}\left(q_{0}\right)-\frac{2}{1+2 q_{0}} F\left(q_{0}\right)+\frac{2 L}{\sqrt{q_{0}\left(1+q_{0}\right)}}=0 .
$$

The left-hand side of (3.1) can be written as

$$
\mathcal{F}\left(L, E, \bar{Q}^{2}, q\right)=\frac{\mathcal{N}\left(L, \bar{M}, \bar{Q}^{2}, q\right)}{\mathcal{D}(a, q)} .
$$

The numerator $\mathcal{N}\left(L, E, \bar{Q}^{2}, q\right)$ contains just one term that depends on $\bar{Q}^{2}$. This dependence is linear with a positive coefficient:

$$
\mathcal{N}\left(a, \bar{M}, \bar{Q}^{2}, q\right)=\mathcal{N}(a, \bar{M}, q)+15 \frac{\pi}{a} \bar{Q}^{2} .
$$

The denominator in (3.2) does not take infinite values. To find solutions of (3.1) for the shape function given by (2.30), we can graph the function

$$
-\mathcal{N}(a, \bar{M}, q) \equiv-\left(512 a^{3} q^{5}+1280 a^{3} q^{4}-96 \bar{M} \pi a q^{2}+1024 a^{3} q^{3}-96 \bar{M} \pi a q+256 a^{3} q^{2}\right)
$$

and see where this function can be equal to a given value $15 \bar{Q}^{\pi} / a$.

To find the maximum allowed $\bar{Q}^{2}$ at which solution of (3.1) still exists, we find the maximum of the function $\mathcal{N}$ for a fixed energy,

$$
\left.\frac{d \mathcal{N}(a, \bar{M}, q)}{d q}\right|_{q=q_{\max }}=0
$$

and the value

$$
\left.\frac{a}{15 \pi} \mathcal{N}(a, \bar{M}, q)\right|_{q=q_{\max }}
$$

defines $\bar{Q}_{\max }^{2}$.

We recall that we work in physical units and use notation (2.34) and (2.33): $\bar{M}=$ $4 G_{5} E / 3 \pi$ and $\bar{Q}^{2}=4 G_{5} Q_{n}^{2} / 3 \pi$. We present the calculation results in figure 5 . 


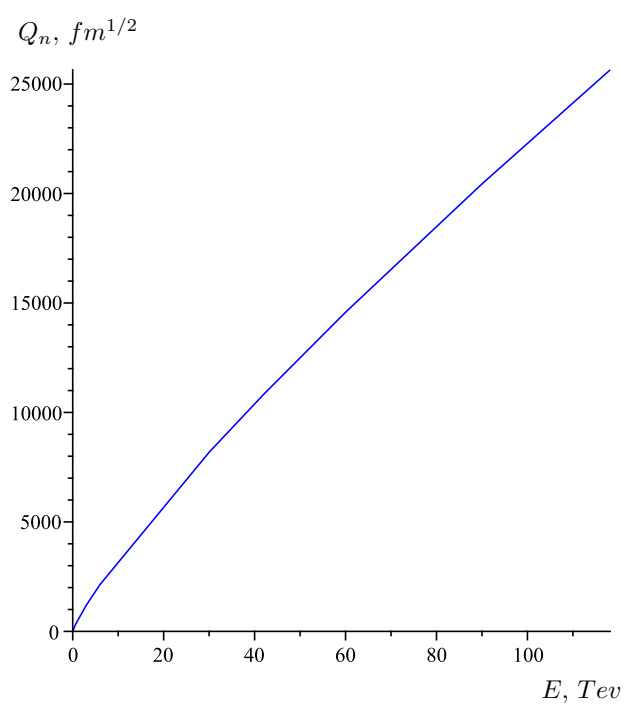

Figure 5. The allowed zone for the TS formation is under the line in the diagram. The plot is constructed using formulas in [34].

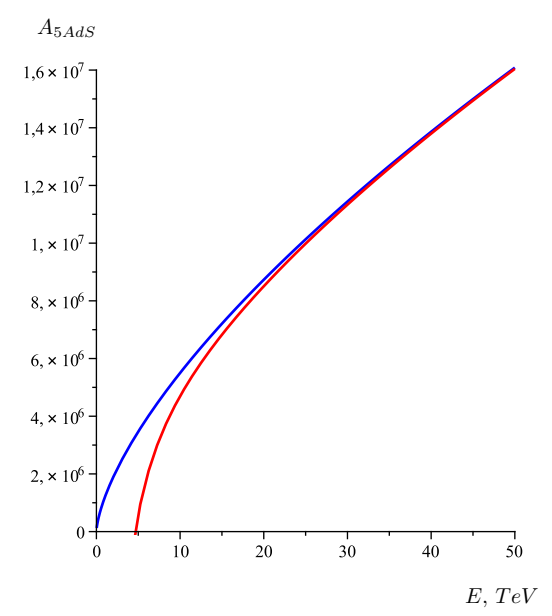

Figure 6. The function $A_{\mathrm{AdS}_{5}}$ at $Q_{n}=0$ (blue line) and $Q_{n}=2 \cdot 10^{6} \mathrm{fm}^{1 / 2}$ (red line).

To estimate corrections to the GYP multiplicity due to a nonzero chemical potential, we use formula (3.17) in [34]. In notation (2.29) and (2.32) used here, the formula has the form

$$
A_{\mathrm{AdS}_{5}} \approx 4 \pi L^{3}\left(\frac{G_{5} E}{L^{2}}\right)^{2 / 3}\left(1-\frac{1}{24}\left(1+\frac{5 Q_{n}^{2}}{E L^{2}}\right)\left(\frac{2 \sqrt{2} L^{2}}{G_{5} E}\right)^{3 / 2}\right)
$$

In figure 6 , we show the entropy $A_{\mathrm{AdS}_{5}}$ for $Q_{n}=0$ and $Q_{n} \neq 0$. The blue line represents $Q_{n}=0$. The red line represents $Q_{n}=2 \cdot 10^{6}$. We see that the deviation from the GYP multiplicity is essential for small energies and is almost negligible for large energies. 


\section{Holographic QGP phase diagram in the wall-on-wall dual model of heavy-ion collisions}

\subsection{Charged wall as a dual model for a heavy ion with a nonzero chemical potential}

We note that the form of the $J_{u u}^{\mathrm{WP}}$ in $(2.49)$ can be obtained by spreading out the energymomentum tensor of an ultrarelativistic point, i.e, $J_{u u}$ in form $(2.22)$ with $\rho(q)$ given by (2.28), over the transverse surface. The Einstein equation for the charged wall (membrane) has the form

$$
\left(\partial_{z}^{2}-\frac{3}{z} \partial_{z}\right) \phi(z)=-16 \pi G_{5}\left(J_{u u}^{\mathrm{WP}}+J_{u u}^{\mathrm{WQ}}(Q, z)\right)
$$

where $J_{u u}^{\mathrm{WP}}$ is given by $(2.49)$ and we assume that $J_{u u}^{\mathrm{WQ}}(Q, z)$ can be obtained by similarly spreading the energy-momentum tensor of the ultrarelativistic charged point $T_{u u}^{\mathrm{p} Q}$ over the transverse surface. In the preceding calculations,

$$
J_{u u}^{\mathrm{WQ}}=\frac{\int_{\mathcal{M}} J_{u u}^{\mathrm{p} Q} \mathcal{D} x_{\perp}}{\int_{\mathcal{M}} \mathcal{D} x_{\perp}}
$$

where the superscript $\mathrm{p} Q$ means the electromagnetic part of the energy-momentum tensor of the charged point particle and $\mathcal{D} x_{\perp}$ means that we integrate over the induced metrics on the orthogonal surface $\mathcal{M}$. For this, we take

$$
J_{u u}^{\mathrm{p} Q}\left(z, z_{0}\right)=\frac{L}{z} \rho^{\mathrm{p} Q}
$$

where $\rho^{\mathrm{p} Q}$ is given by (2.31) and we integrate over all transverse coordinates according to our prescription (4.2),

$$
J_{u u}^{\mathrm{p} Q, \mathrm{II}}=\frac{(L / z) \int_{0}^{\infty}\left(\rho^{\mathrm{p} Q}(q) L^{2} / 2 z_{0}^{2}\right) d r^{2}}{\int_{0}^{\infty}\left(r L^{2} / z_{0}^{2}\right) d r} .
$$

The result is

$$
J_{u u}^{\mathrm{p} Q}=\mathcal{X} \mathcal{J}
$$

where

$$
\begin{aligned}
& \mathcal{J}=\frac{64}{3} z z_{0}\left(1-\frac{z_{0}^{6}-3 z^{2} z_{0}^{4}-3 z^{4} z_{0}^{2}+z^{6}}{\left|z_{0}^{2}-z^{2}\right|^{3}}\right), \\
& \mathcal{X}=\frac{5}{256} \frac{Q_{n}^{2}}{\pi z L^{6}}=\frac{5}{256} \frac{Q^{2}}{z L^{6}} .
\end{aligned}
$$

We see a divergence at $z=z_{0}$, as should be for the energy-momentum tensor of a charged plane. We introduce regularization by adding the $\epsilon$ factor in the denominator. 


\subsection{Charged wall-on-wall collision as a dual model for heavy-ion collisions with a nonzero chemical potential}

To find the TS formation condition in the wall-wall collision, we must solve the Einstein equation

$$
\begin{aligned}
\left(\partial_{z}^{2}-\frac{3}{z} \partial_{z}\right) \phi(z) & =-16 \pi G_{5}\left(J_{u u}^{\mathrm{WP}}(z)+J_{u u}^{\mathrm{WQ}}(Q, z)\right), \\
J_{u u}^{\mathrm{WP}}(z) & =\frac{E}{L^{2}} \frac{z_{0}^{3}}{L^{3}} \delta\left(z-z_{0}\right), \\
J_{u u}^{\mathrm{WQ}}(Q, z) & =\frac{128 \mathcal{X}}{3} z z_{0} \frac{z^{4}\left(-z^{2}+3 z_{0}^{2}\right) \theta\left(z_{0}-z\right)+z_{0}^{4}\left(-3 z^{2}+z_{0}^{2}\right) \theta\left(z-z_{0}\right)}{\left(-z^{2}+z_{0}^{2}+\epsilon^{2}\right)^{3}},
\end{aligned}
$$

with the boundary conditions

$$
\begin{aligned}
\phi\left(z_{a}\right)=\phi\left(z_{b}\right) & =0, & \phi\left(z_{0}+0\right) & =\phi\left(z_{0}-0\right), \\
\psi^{\prime}\left(z_{a}\right) \frac{z_{a}}{L} & =2, & \psi^{\prime}\left(z_{b}\right) \frac{z_{b}}{L} & =-2,
\end{aligned}
$$

where $z_{a}$ and $z_{b}$ are the boundaries of the TS and $\psi$ is related to $\phi(z)$ as

$$
\phi(z)=\frac{z}{L} \psi
$$

We seek a solution of the Einstein equation with a charged source in the form of the sum of the "neutral" solution and a correction proportional to $Q^{2}$ :

$$
\phi=\phi_{\mathrm{n}}+\phi_{q},
$$

where $\phi_{\mathrm{n}}$ denotes the solution in the neutral case.

As in the neutral case, it is convenient to consider the domains $z<z_{0}$ and $z>z_{0}$ separately,

$$
\phi_{q}= \begin{cases}\phi_{q z_{0}>z}, & z_{0}>z \\ \phi_{q z>z_{0}}, & z>z_{0}\end{cases}
$$

and we have

$$
\begin{aligned}
& \left(\partial_{z}^{2}-\frac{3}{z} \partial_{z}\right) \phi_{q z_{0}>z}=-16 \pi G_{5} \mathcal{X} \frac{128}{3} z z_{0} \frac{z^{4}\left(-z^{2}+3 z_{0}^{2}\right)}{\left(-z^{2}+z_{0}^{2}+\epsilon^{2}\right)^{3}}, \quad z_{0}>z \\
& \left(\partial_{z}^{2}-\frac{3}{z} \partial_{z}\right) \phi_{q z>z_{0}}=-16 \pi G_{5} \mathcal{X} \frac{128}{3} z z_{0} \frac{z_{0}^{4}\left(-3 z^{2}+z_{0}^{2}\right)}{\left(-z^{2}+z_{0}^{2}-\epsilon^{2}\right)^{3}}, \quad z>z_{0} .
\end{aligned}
$$

Solutions of (4.16) and (4.17) can be represented as

$$
\begin{aligned}
& \psi_{q z_{0}>z}=z^{3} C_{1}+\frac{C_{2}}{z}-\frac{N L z_{0} z^{3}}{4\left(-z^{2}+z_{0}^{2}+\epsilon^{2}\right)}, \quad z_{0}>z, \\
& \psi_{q z>z_{0}}=\frac{C_{3}}{z}+z^{3} C_{4}+\frac{N L z_{0}^{5}}{4 z\left(-z^{2}+z_{0}^{2}+\epsilon^{2}\right)}, \quad z>z_{0},
\end{aligned}
$$


where $N=40 \pi G_{5} Q^{2} / 3 L^{6}$. The first two terms in (4.18) and (4.19) are the solution of Lin-Shuryak equation (55) in [29]. If we assume that they satisfy condition (4.11), i.e., $\psi_{\mathrm{n}}\left(z_{a}\right)=\psi_{\mathrm{n}}\left(z_{b}\right)=0$ and $\psi_{\mathrm{n} a}\left(z_{0}\right)=\psi_{\mathrm{n} b}\left(z_{0}\right)$, then we obtain [77]

$$
\Psi_{\mathrm{n}}= \begin{cases}\psi_{\mathrm{n} a}=C\left(\frac{z^{3}}{z_{a}^{3}}-\frac{z_{a}}{z}\right), & C=-\frac{4 \pi G_{5} E}{L^{4}} \frac{z_{0}^{4} z_{b} / z_{b}^{4}-1}{\left(z_{b}^{4}-z_{a}^{4}\right) / z_{a}^{3} z_{b}^{3}}, \quad z<z_{0}, \\ \psi_{\mathrm{n} b}=D\left(\frac{z^{3}}{z_{b}^{3}}-\frac{z_{b}}{z}\right), & D=-\frac{4 \pi G_{5} E}{L^{4}} \frac{z_{0}^{4} z_{a} / z_{a}^{4}-1}{\left(z_{b}^{4}-z_{a}^{4}\right) / z_{a}^{3} z_{b}^{3}}, \quad z_{0}<z .\end{cases}
$$

In the neutral case, we can find $z_{a}$ and $z_{b}$ from the second condition $\psi_{\mathrm{na}}^{\prime}\left(z_{a}\right) z_{a} / L=2$ and $\psi_{\mathrm{n} b}^{\prime}\left(z_{b}\right) z_{b} / L=-2$, where $z_{a}$ and $z_{b}$ are the boundaries of the TS.

For (4.18) and (4.19), choosing

$$
\begin{array}{ll}
C_{1}=\frac{N L z_{0}}{4\left(z_{a}^{2}-z_{0}^{2}\right)}, & C_{2}=0, \\
C_{3}=\frac{N L z_{0}^{5}}{4\left(z_{b}^{2}-z_{0}^{2}\right)}, & C_{4}=0,
\end{array}
$$

we obtain

$$
\begin{cases}\psi_{a q}=-\frac{N L z_{0} z^{3}}{4} \frac{-z_{a}^{2}+z^{2}-\epsilon^{2}}{\left(-z^{2}+z_{0}^{2}+\epsilon^{2}\right)\left(-z_{a}^{2}+z_{0}^{2}\right)}, & z<z_{0} \\ \psi_{b q}=\frac{N L z_{0}^{5}}{4 z} \frac{-z_{b}^{2}+z^{2}+\epsilon^{2}}{\left(-z^{2}+z_{0}^{2}-\epsilon^{2}\right)\left(-z_{b}^{2}+z_{0}^{2}\right)}, & z_{0}<z .\end{cases}
$$

We note that the constructed solution automatically satisfies the condition $\psi\left(z_{a}\right)=\psi\left(z_{b}\right)=$ 0 . Condition (4.12) gives

$$
\begin{gathered}
-\frac{8 \pi G_{5} E\left(z_{0}^{4}-z_{b}^{4}\right) z_{a}^{3}}{L^{5}\left(z_{b}^{4}-z_{a}^{4}\right)}-\frac{N}{4} \frac{z_{0} z_{a}^{5}}{\left(-z_{a}^{2}+z_{0}^{2}\right)^{2}}=1, \\
-\frac{8 \pi G_{5} E\left(z_{0}^{4}-z_{a}^{4}\right) z_{b}^{3}}{L^{5}\left(z_{b}^{4}-z_{a}^{4}\right)}+\frac{N}{4} \frac{z_{0}^{5} z_{b}}{\left(-z_{b}^{2}+z_{0}^{2}\right)^{2}}=-1 .
\end{gathered}
$$

These equations do not have analytic solutions, and we treat them numerically.

The roots of system (4.24), (4.25) cannot be found analytically, because these equations are equivalent to polynomial equations in $z_{a}$ and $z_{b}$ of a high degree $(>4)$. We therefore take $z_{0}=L$ and numerically analyze the system

$$
\begin{aligned}
& F_{a} \equiv-\frac{8 \pi G_{5} E\left(z_{0}^{4}-z_{b}^{4}\right) z_{a}^{3}}{z_{0}^{5}\left(z_{b}^{4}-z_{a}^{4}\right)}-\frac{10}{3} \frac{\pi G_{5} Q^{2}}{z_{0}^{6}} \frac{z_{0} z_{a}^{5}}{\left(-z_{a}^{2}+z_{0}^{2}\right)^{2}}=1 \\
& F_{b} \equiv-\frac{8 \pi G_{5} E\left(z_{0}^{4}-z_{a}^{4}\right) z_{b}^{3}}{z_{0}^{5}\left(z_{b}^{4}-z_{a}^{4}\right)}+\frac{10}{3} \frac{\pi G_{5} Q^{2}}{z_{0}^{6}} \frac{z_{0}^{5} z_{b}}{\left(-z_{b}^{2}+z_{0}^{2}\right)^{2}}=-1
\end{aligned}
$$

To show the movement of the roots of (4.26) and (4.27), we assume that $z_{b}$ for a given $Q$ is already known and represent the function $F_{a}\left(z_{a}, z_{b}\right)$ as a function of $z_{a}$ in figure 7 . Similarly, assuming that $z_{a}$ is already known, we represent the function $F_{b}\left(z_{a}, z_{b}\right)$ as a function of $z_{b}$ in figure 8 .

In figure 9 , we show the charge flows of the roots. Different lines correspond to different energies. We see that the flows go to $z_{0}$ and reach the line $z=z_{0}$ for $Q=Q_{\mathrm{cr}}$. In figure 10, we draw the corresponding flow for physical parameters. 


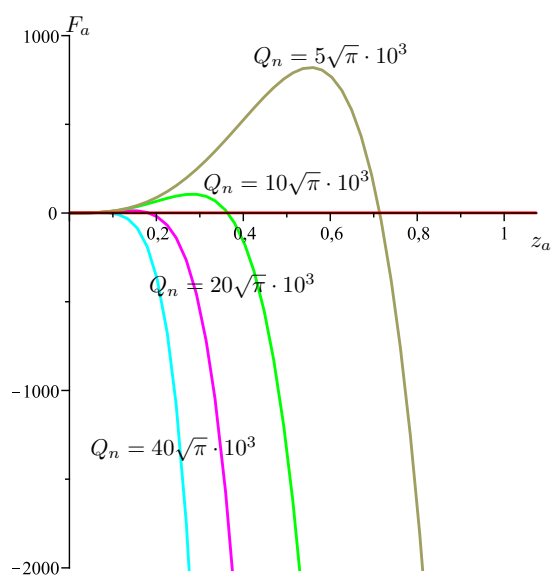

(a)

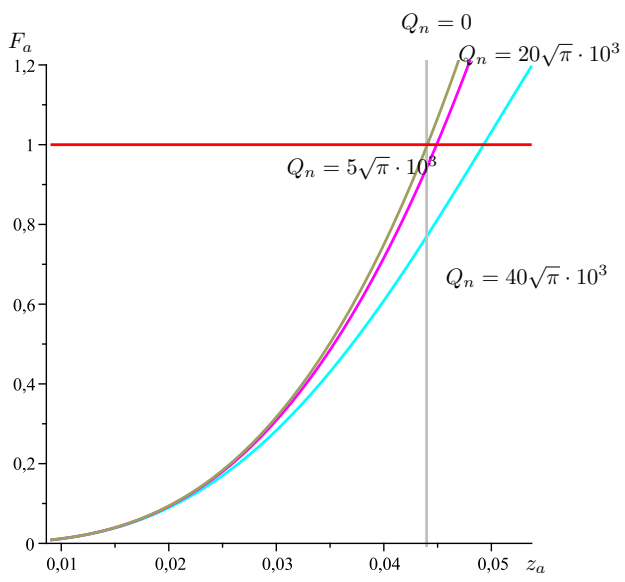

(b)

Figure 7. (a) Plot of $F_{a}\left(z_{a}, z_{b}\right)$ as a function of $z_{a}$ for a fixed $z_{b}$ (which is taken to be a root $z_{b}=z_{b}(E)$ of system of equations $\left.(4.27),(4.28)\right)$ near the root $z_{a}=z_{a}(E)$ at $E=118.2 \mathrm{TeV}$. (b) Enlargement of the region of small $F_{a}$ and small $z_{a}$.

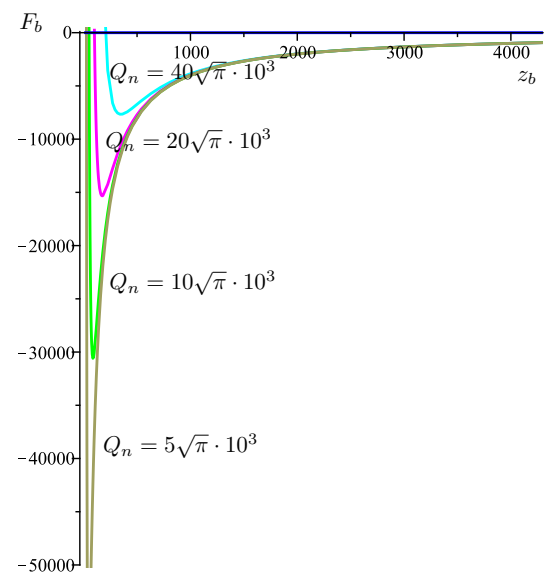

(a)

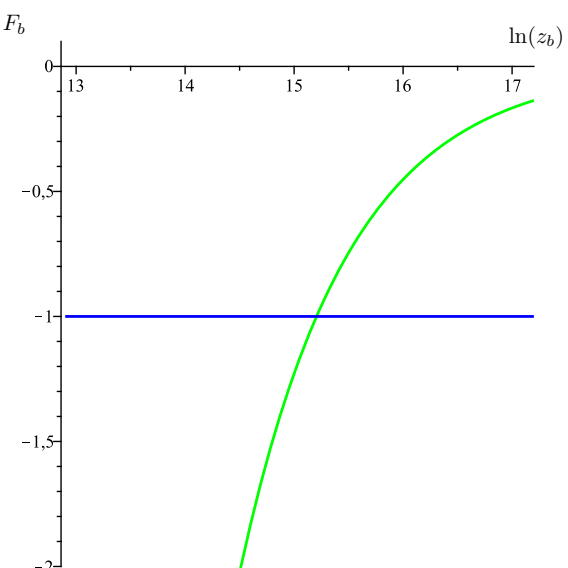

(b)

Figure 8. (a) Plot of $F_{b}\left(z_{a}, z_{b}\right)$ as a function of $z_{b}$ for a fixed $z_{a}$ (which is taken to be a root $z_{a}=z_{a}(E)$ of system of equations (4.27), (4.28)) near the root $z_{b}=z_{b}(E)$ at $E=118.2 \mathrm{TeV}$. (b) Logarithmic enlargement of the region of small $F_{b}$ and large $z_{b}$.

\subsection{Comparison of results}

It is interesting to compare the phase diagrams of the energy (temperature) $E$ versus the charge (chemical potential) $Q$ corresponding to the pointlike charge and the spread charge. The results of these calculations are collected in the table below and presented in figure 11 . It can be seen that these two phase diagrams are almost the same.

\begin{tabular}{|c|c|c|c|c|c|c|c|c|c|c|}
\hline$E, \mathrm{TeV}$ & 118.2 & 60 & 30 & 6 & 3 & 0.6 & 0.06 & 0.03 & 0.0003 & 0.00025 \\
\hline$Q_{\text {cr, point }}$ & 25649.6 & 14577.2 & 8180.6 & 2138.7 & 1199.9 & 313.3 & 45.6 & 25.4 & 0.43 & 0.37 \\
$Q_{\text {cr, wall }}$ & 47500 & 27000 & 15170 & 3950 & 2220 & 570 & 80 & 40 & 0.15 & 0 \\
\hline
\end{tabular}




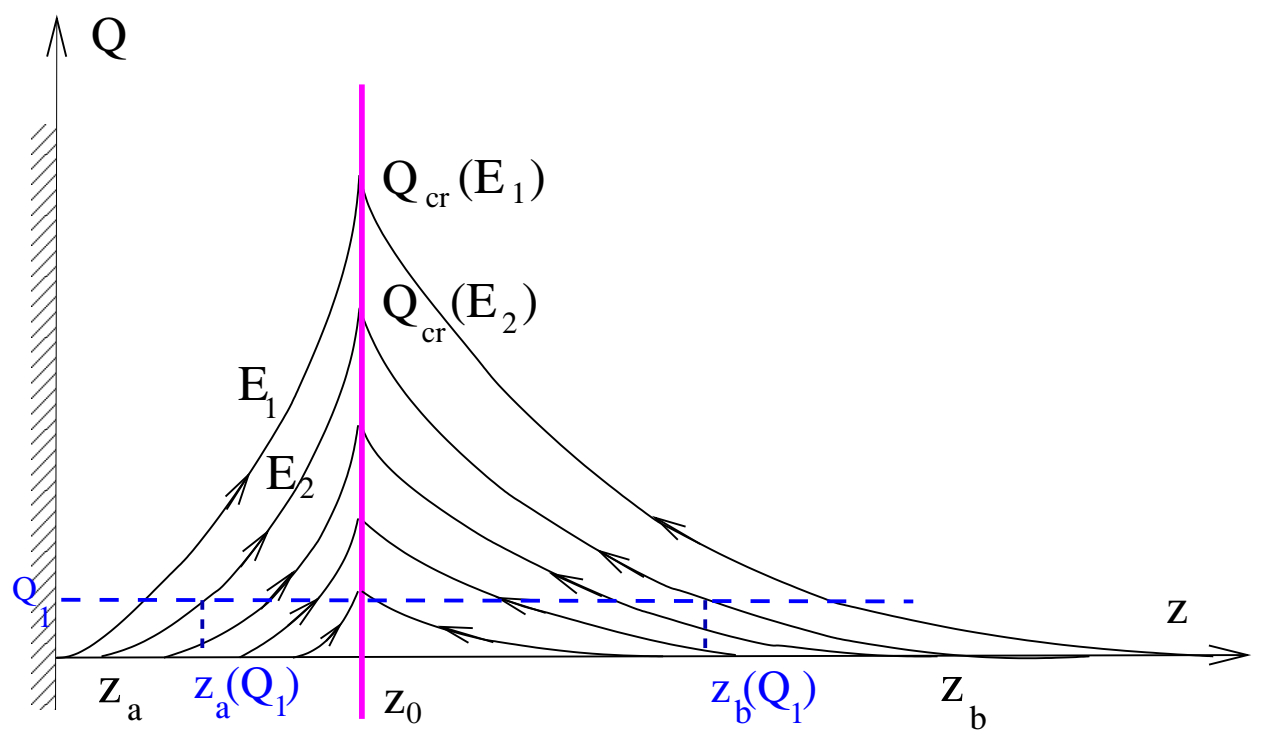

Figure 9. Schematic picture of charge flows: The magenta solid line shows the position of the wall. The positions of the points $z_{a}(Q)$ and $z_{b}(Q)$ move to the point $z=z_{0}$ as $Q$ increases. The segment $\left[z_{a}(Q), z_{b}(Q)\right]$ shrinks to zero as $Q \rightarrow Q_{\mathrm{cr}}(E)$.

It is evident from figure 11 that the red and blue lines intersect. We present the intersection in the natural and logarithmic scales in figure 12.

\subsection{The square TS calculation}

Following [29], we calculate the lower bound of the entropy as the "TS area" per unit square of the wall ${ }^{3}$ using the formula

$$
\begin{aligned}
& S=\frac{2 A}{4 G_{5}}=\frac{\int \sqrt{g} d z d^{2} x_{\perp}}{2 G_{5}}, \\
& s \equiv \frac{S}{\int d^{2} x_{\perp}}=\frac{L^{3}}{4 G_{5}}\left(\frac{1}{z_{a}^{2}}-\frac{1}{z_{b}^{2}}\right) .
\end{aligned}
$$

In the absence of transverse dependence, we ignore $x_{\perp}^{2}$ in (4.28). Equation (4.29) measures the entropy per transverse area.

The TS decreases as the charge increases. The corresponding graphical representations are in figure 13.

In figure 14, we show the entropy per volume given by (4.29) as a function of energy for different $Q$. This plot is similar to the plot presented in figure 6 . It can be seen that the influence of the chemical potential on the multiplicity is essential for small energies and is almost negligible for large energies.

In the work [28] it has been suggested to place UV and IR cut-offs in order to reduce the multiplicities. Later, in the work [38] it was realized that most of the entropy comes

\footnotetext{
${ }^{3}$ We write "TS area" in quotation marks because the TS in the strict sense must be smooth and compact. In our case, it is nonsmooth and does not have finite area, and these properties can be assumed only after regularization.
} 


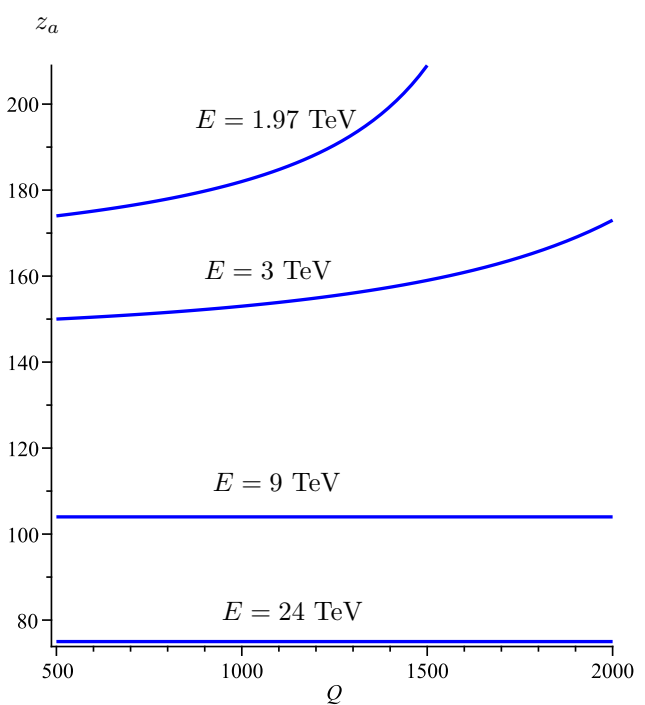

(a)

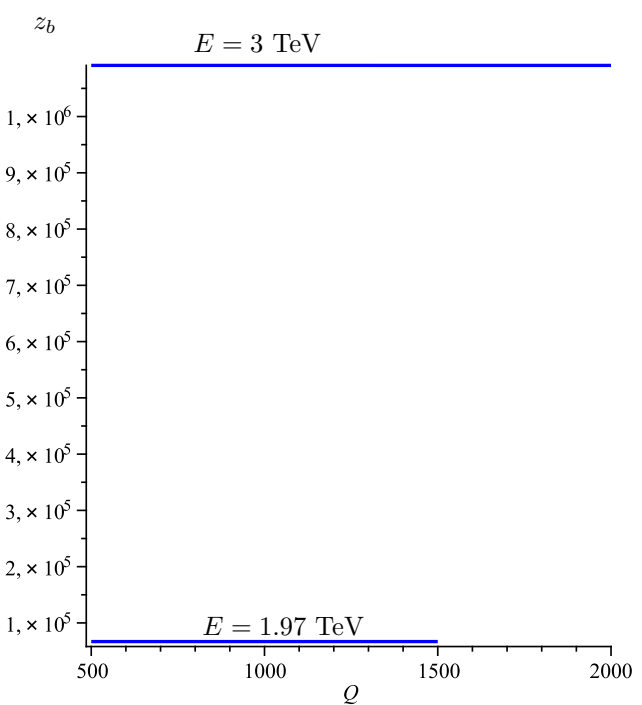

(b)

Figure 10. (a) Charge flows of the root $z_{a}(Q)$ for $E=1.97,3,9,24 \mathrm{TeV}$. (b) Charge flows of the roots $z_{b}(Q)$ for $E=1.97,3 \mathrm{TeV}$.

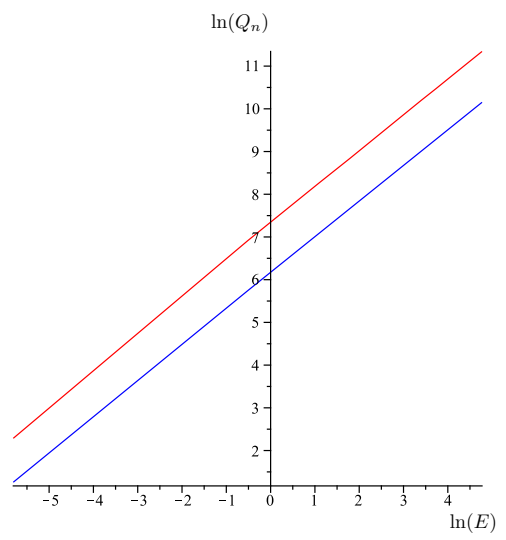

(a)

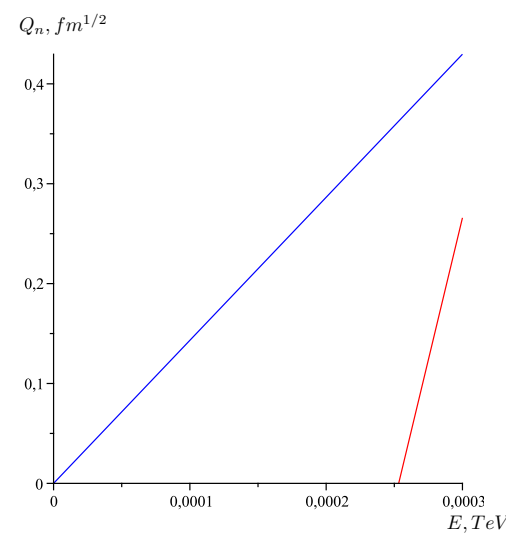

(b)

Figure 11. (a) Phase diagram of $\log Q_{n}$ versus $\log E$ at large $E$. (b) Phase diagram of $E$ versus $Q_{n}$ for small $E$ and small $Q_{n}$. The blue lines correspond to the pointlike charge, and the red lines correspond to the spread charge. The zones above the lines are forbidden for $\mathrm{BH}$ production for the corresponding $E$ and $Q$.

from the UV part of the trapped surface (small z) and hence the IR cut-off is not very important. In the case of the wall shock wave collisions the main contribution comes from the region located near the wall $\left(z=z_{0}\right)$. Performing the IR cut-off by removing $z$ larger than $z_{b}(E)$ we do not change the multiplicity. This is evident from the formula (4.29). But the UV cut-off can affect the multiplicity. If we remove $z$ smaller then $z_{a}(E)$ we do change the multiplicity significantly, see figure 9 .

As to the point source shock waves the explicit formulae are more complicated but one can expect the similar results. 


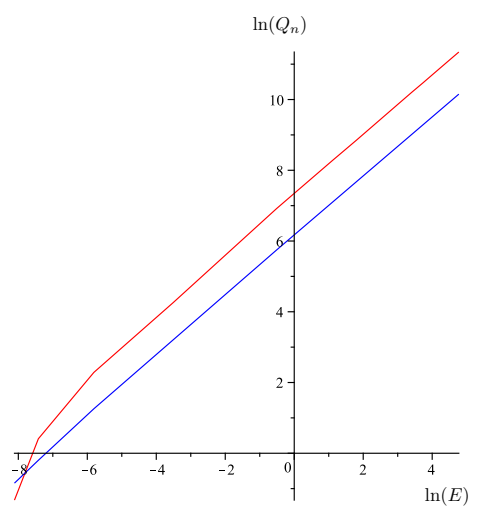

(a)

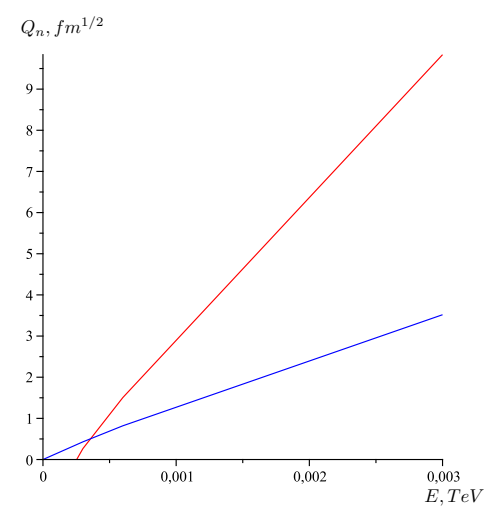

(b)

Figure 12. The intersection of the two diagrams in (a) logarithmic and (b) natural scales.

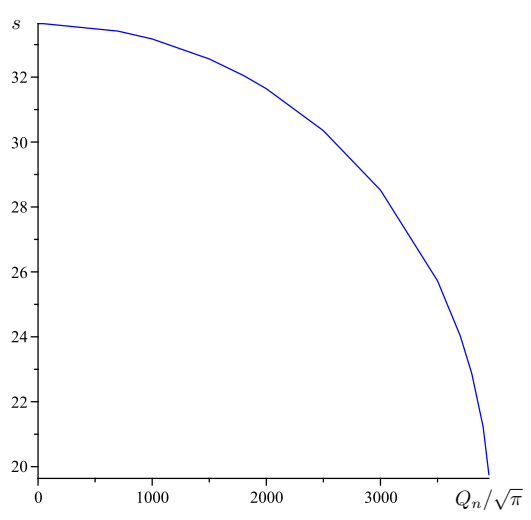

(a)

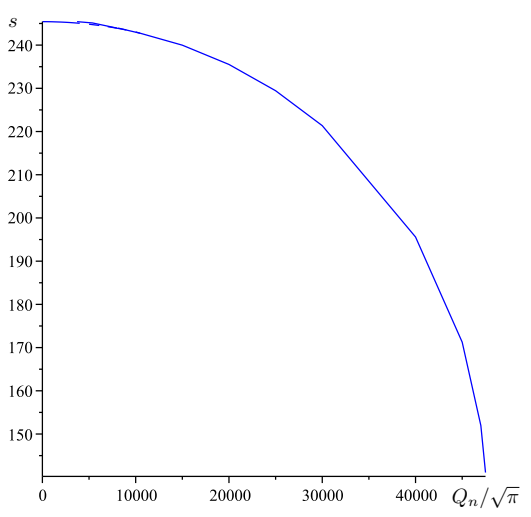

(b)

Figure 13. Dynamics of the TS area $s\left(Q_{n} / \sqrt{\pi}\right)$ at (a) $E=6 \mathrm{TeV}$ and (b) $E=118.2 \mathrm{TeV}$.

\subsection{Remarks about the regularization}

The regularized version of the the function $\psi$ is

$$
\psi_{\text {reg }}=\psi_{a}(z) \Gamma_{1}+\psi_{b}(z) \Gamma_{2}
$$

where $\psi_{a}(z)$ and $\psi_{b}(z)$ define the function $\psi$ without regularization:

$$
\psi=\psi_{a}(z) \Theta\left(z_{0}-z\right)+\psi_{b}(z) \Theta\left(z-z_{0}\right)
$$

where

$$
\begin{aligned}
& \psi_{a}(z)=-\frac{4 G \pi E\left(z_{0}^{4} / z_{b}^{4}-1\right) z_{b}^{4} z_{a}^{3}\left(z^{3} / z_{a}^{3}-z_{a} / z\right)}{L^{4}\left(z_{b}^{4}-z_{a}^{4}\right)}-\frac{10}{3} \frac{Q^{2} G \pi z_{0} z^{3}\left(-z_{a}^{2}+z^{2}\right)}{L^{5}\left(-z^{2}+z_{0}^{2}\right)\left(-z_{a}^{2}+z_{0}^{2}\right)} \\
& \psi_{b}(z)=-\frac{4 G \pi E\left(z_{0}^{4} / z_{a}^{4}-1\right) z_{a}^{4} z_{b}^{3}\left(z^{3} / z_{b}^{3}-z_{b} / z\right)}{L^{4}\left(z_{b}^{4}-z_{a}^{4}\right)}+\frac{10}{3} \frac{Q^{2} G \pi z_{0}^{5}\left(-z_{b}^{2}+z^{2}\right)}{L^{5} z\left(-z^{2}+z_{0}^{2}\right)\left(-z_{b}^{2}+z_{0}^{2}\right)},
\end{aligned}
$$




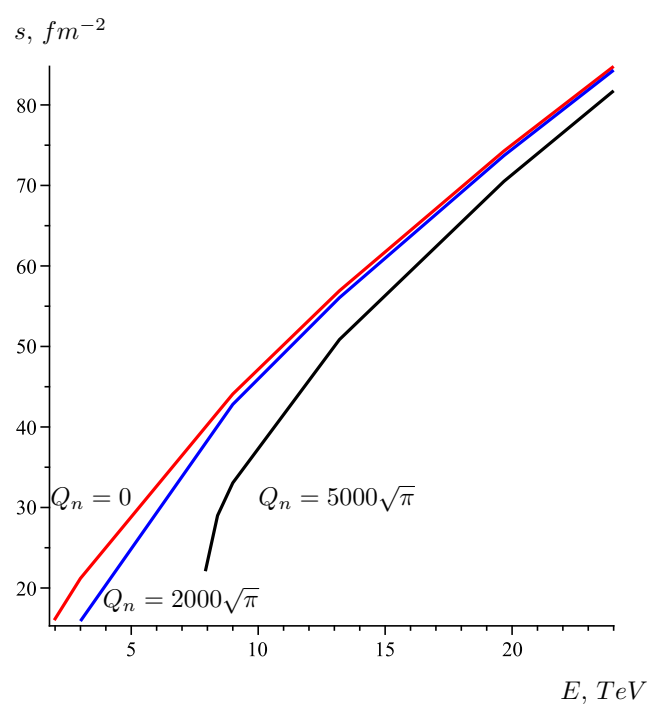

Figure 14. Entropy per volume as a function of energy for different $Q$ : red line, $Q_{n}=0 \mathrm{fm}^{1 / 2}$; blue line, $Q_{n}=2000 \sqrt{\pi} \mathrm{fm}^{1 / 2}$; black line, $Q_{n}=5000 \sqrt{\pi} \mathrm{fm}^{1 / 2}$.

and

$$
\begin{aligned}
& \Gamma_{1}=\frac{\arctan \left(R\left(z_{0}-z\right)\right)^{3}}{\pi}+\frac{1}{2}, \\
& \Gamma_{2}=\frac{\arctan \left(R\left(z-z_{0}\right)\right)^{3}}{\pi}+\frac{1}{2} .
\end{aligned}
$$

We must now impose conditions (2.52) on the regularized functions

$$
\left.\frac{z_{a}}{2 L} \frac{d}{d z} \psi_{\mathrm{reg}}\right|_{z=\tilde{z}_{a}}=1
$$

But it is difficult to find $\tilde{z}_{a}$ from condition (4.34). Instead of finding $\tilde{z}_{a}$ from condition (4.34), we propose to use a regularization that does not change $z_{a}$ found from formal conditions (2.52). We can verify that the formal $z_{a}$ in fact also satisfies the regularized condition if the regularization is sufficiently smooth. We therefore take $z_{a}$ and substitute it in the left-hand side of regularized condition (4.34). We define

$$
\begin{aligned}
& \left.F_{a, \text { reg }}\right|_{z=z_{a}}=\left.\frac{z_{a}}{2 L}\left(\frac{d \psi_{a}}{d z} \Gamma_{1}+\frac{d \psi_{b}}{d z} \Gamma_{2}\right)\right|_{z=z_{a}} \approx 1, \\
& \left.F_{b, \text { reg }}\right|_{z=z_{b}}=\left.\frac{z_{b}}{2 L}\left(\frac{d \psi_{a}}{d z} \Gamma_{1}+\frac{d \psi_{b}}{d z} \Gamma_{2}\right)\right|_{z=z_{b}} \approx-1 .
\end{aligned}
$$

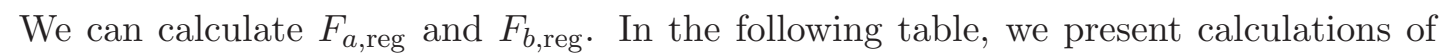
$F_{a, r e g}$ and $F_{b, r e g}$ at $R=10^{4}$ for a wide range of the theory parameter. It is obvious from 
the table that $F_{a} \approx 1$ and $F_{b} \approx-1$.

\begin{tabular}{|c|c|c|c|c|c|}
\hline$E, \mathrm{TeV}$ & $Q=Q_{n} / \sqrt{\pi}, \mathrm{fm}^{1 / 2}$ & $z_{a}, \mathrm{fm}$ & $z_{b}, \mathrm{fm}$, & $F_{a}$ & $F_{b}$ \\
\hline 118.2 & 40000 & 0.04928014740 & $4.015208864 \cdot 10^{6}$ & 0.99997 & -1.00000 \\
3 & 15000 & 0.08847525298 & $1.019088359 \cdot 10^{6}$ & 1.00000 & -1.00000 \\
0.03 & 40 & 0.7861838575 & 1017.792389 & 1.00000 & -1.00000 \\
\hline
\end{tabular}

\section{Conclusion}

\subsection{Summary}

We have used a holographic dual model for a heavy-ion collision to construct the phase diagram of the QGP formed at a very early stage just after the collision. In this dual model, colliding ions are described by charged gravitational shock waves. Points on the phase diagram correspond to the QGP or hadronic matter with given temperatures and chemical potentials. The QGP phase in dual terms is related to the case where the collision of shock waves leads to the formation of a TS. Hadronic matter and other confined states correspond to the absence of a TS after the collision.

The multiplicity of the ion collision process was estimated in the dual language as the TS area. We showed that a nonzero chemical potential reduces the multiplicity. To plot the phase diagram, we used two different dual models of colliding ions. The first model uses the point shock waves, and the second uses the wall shock waves. We found that the results agree qualitatively.

We paid special attention to the regularization procedure for the calculations for wall shock waves. On one hand, these calculations are essentially simpler technically. On the other hand, this approach, strictly speaking, is incorrect and requires a regularization. We showed that a natural regularization does exist. Moreover, the proposed regularization does not make the calculations more complicated compared with the naive (direct) calculations. This opens new possibility for simple calculations for wall shock waves carrying nontrivial matter charges.

\subsection{Further directions}

Head-on collisions of charged point shock waves have only two parameters. In the dual language, they correspond to the energy and the chemical potential per nucleus. Off-center collisions are also specified by the impact parameter, and the change of this parameter can be associated with a dual change from "nonthermal" peripheral to "thermal" central collisions [29]. But this is still an oversimplification of the problem. The physics of heavyion collisions in the RHIC is richer, and as indicated in [29, 77], the rapid equilibration and hydrodynamic behavior experimentally observed at the RHIC for collisions of two heavy ions such as $\mathrm{Au}-\mathrm{Au}$ does not occur for deuteron-Au collisions at the same rapidity. It is perhaps too naive to believe that the simplest shock wave related by a boost to the Schwarzschild BH in the AdS space can mimic the nuclear matter in the colliders. But this 
simple shock wave in fact reproduces the interaction of a relativistic quark with gravity and can therefore be regarded as a simplest candidate for mimicking nuclear matter in the holographic conjecture. We can try to associate different nuclei with different forms of shock waves. In this context, we recall that the form of the shock wave follows from the eikonal approximation of the gravity-quark interaction in five dimensions [39, 78, 79]. The presence of an electromagnetic field or other fields and also any improvements of the eikonal approximation certainly changes the form of the shock waves, and it would be interesting to see the holographic consequences of this consideration.

The lower bound obtained for $N_{\mathrm{ch}}$ scales as $s_{N N}^{1 / 3}$, which is a faster energy dependence than the $s_{N N}^{1 / 4}$ scaling predicted by the Landau model [50] and largely satisfied by the data. If we have an a priori restriction on the allowed energy, then we can fit constants to ensure that the experimental data are above the AdS bound. We note that taking the chemical potential into account allows increasing the permitted energy. But we cannot expect too much from the chemical potential corrections. The relevant chemical potential for the baryon number is not expected to be large, i.e., $\mu_{\mathrm{B}} \sim 30 \mathrm{MeV}$ or $\mu_{\mathrm{B}} / \mathrm{T} \sim 0.15$ for recent experiments at the RHIC [80], and any effects will therefore be limited. Nevertheless, as mentioned in the text, the relation between the chemical potential value and the fivedimensional charge value is at our disposal, and we can assume that they have a huge ratio.

It would also be interesting to try to use plane gravitational waves in $\mathrm{AdS}_{5}$ to describe nonperturbative stages in the gauge theories and to use collisions of these waves to describe the QGP formed in heavy-ion collisions. In the planar case, the Chandrasekhar-FerrariXanthopoulos duality between colliding plane gravitational waves and the Kerr BH solution was used as a model of $\mathrm{BH}$ formation [81]. It would be interesting to generalize this duality to the AdS case. This may yield a new insight into the possible dependence of multiplicities on the rapidity.

\section{Acknowledgments}

The authors thank S. Yu. Vernov and I. V. Volovich for the helpful discussions. One of the authors (A. A. B.) is grateful to J. Zaanen, K. Schalm, A. Parnachev, and M. Cubrovic for the illuminating conversations about AdS holography. This work is supported in part by the Russian Foundation for Basic Research (Grant No. 11-01-00894-a). The work of A. A. B. is also supported by the Dynasty Foundation and the Dutch Foundation for Fundamental Research on Matter (FOM). The work of E. O. P. is supported in part by the Russian Ministry of Education and Science (Grant No. 14.740.12.0846).

\section{A Metric of an ultrarelativistic charge in an AdS background}

The metric of an ultrarelativistic charge in the flat background was obtained in [82-84] by applying the Aichelburg-Sexl boost [85] to the $D$-dimensional RN space-time. The same procedure can be applied to the RN-AdS BH metric [34]. These calculations generalize the results in $[60,61,63,64,82,83]$ (see more references in [34]). A simpler way to obtain the answer is to consider the $\mathrm{RN}-(\mathrm{A}) \mathrm{dS}$ metric in spherical static (Schwarzschild) 
coordinates (2.8), (2.9), expand it in $M$ and $Q^{2}$,

$$
d s^{2}=d s_{\text {AdS }}^{2}+\left(\frac{2 M}{R^{D-3}}-\frac{Q^{2}}{R^{2(D-3)}}\right) d T^{2}+\frac{1}{\left(1+\Lambda R^{2} / 3\right)^{2}}\left(\frac{2 M}{R^{D-3}}-\frac{Q^{2}}{R^{2(D-3)}}\right) d R^{2},
$$

and rewrite (A.1) in plane coordinates (in which the form of the boost is more transparent). The plane coordinates satisfy the relation

$$
-Z_{0}^{2}+Z_{1}^{2}+Z_{2}^{2}+\cdots+Z_{D-1}^{2}-Z_{D}^{2}=-a^{2}
$$

and are related to the Schwarzschild coordinates as

$$
\begin{aligned}
Z_{0} & \equiv \sqrt{a^{2}+R^{2}} \sin (T / a), \\
Z_{D} & \equiv \sqrt{a^{2}+R^{2}} \cos (T / a), \\
Z_{1} & \equiv R \cos \theta_{1}, \\
Z_{2} & \equiv R \sin \theta_{1} \cos \theta_{2}, \ldots, \\
Z_{D-1} & =R \sin \theta_{1} \sin \theta_{2} \cdots \sin \theta_{D-2} .
\end{aligned}
$$

In the plane coordinates, metric (A.1) is

$$
d s^{2}=d s_{0}^{2}+d s_{p}^{2}
$$

where $d s_{0}^{2}$ is the AdS metric and the perturbation $d s_{p}^{2}$ has the form

$$
d s_{p}^{2}=G_{00} d Z_{0}^{2}+G_{D D} d Z_{D}^{2}+G_{0 D} d Z_{0} d Z_{D}
$$

where

$$
G_{M N}=\chi\left(Z_{0}^{2}, Z_{D}^{2}, M, Q\right) \cdot g_{M N}\left(Z_{0}^{2}, Z_{D}^{2}\right)
$$

and the nonzero components of $g_{M N}$ and the overall factor $\chi\left(Z_{0}^{2}, Z_{D}^{2}, M, Q\right)$ are given by

$$
\begin{aligned}
g_{00} & =-a^{2} Z_{D}^{2}+Z_{0}^{2} Z_{D}^{2}+Z_{D}^{4}+a^{2} Z_{0}^{2}, \\
g_{D D} & =-a^{2} Z_{0}^{2}+Z_{0}^{4}+Z_{D}^{2} Z_{0}^{2}+a^{2} Z_{D}^{2}, \\
g_{0 D} & =-2\left(-2 a^{2}+Z_{0}^{2}+Z_{D}^{2}\right) Z_{0} Z_{D}, \\
\chi & =\frac{a^{2}}{\left(Z_{D}^{2}+Z_{0}^{2}\right)^{2}}\left(\frac{2 M}{\left(-a^{2}+Z_{0}^{2}+Z_{D}^{2}\right)^{(D-1) / 2}}-\frac{Q^{2}}{\left(-a^{2}+Z_{0}^{2}+Z_{D}^{2}\right)^{D-2}}\right) .
\end{aligned}
$$

Performing a boost in the $Z_{1}$ direction,

$$
\begin{aligned}
& Z_{0}=\gamma\left(Y_{0}+v Y_{1}\right), \quad \gamma \equiv\left(1-v^{2}\right)^{-1 / 2}, \\
& Z_{1}=\gamma\left(v Y_{0}+Y_{1}\right), \\
& Z_{2}=Y_{2}, \quad \ldots, \quad Z_{D}=Y_{D}
\end{aligned}
$$


and rescaling,

$$
\begin{aligned}
M & =\bar{M} \sqrt{1-v^{2}} \equiv \bar{M} / \gamma, \\
Q^{2} & =\bar{Q}^{2} \sqrt{1-v^{2}} \equiv \bar{Q}^{2} / \gamma
\end{aligned}
$$

we write the first-order deformation of the metric in the form

$$
\begin{aligned}
d s_{p}^{2}= & \gamma G_{00}\left(\gamma^{2}\left(Y_{0}+v Y_{1}\right)^{2}, Y_{D}^{2}\right) d\left(Y_{0}+v Y_{1}\right)^{2} \\
& +G_{D D}\left(\gamma^{2}\left(Y_{0}+v Y_{1}\right)^{2}, Y_{D}^{2}\right) d\left(Y_{0}+v Y_{1}\right) d Y_{D} \\
& +\frac{1}{\gamma} G_{D D}\left(\gamma^{2}\left(Y_{0}+v Y_{1}\right)^{2}, Y_{D}^{2}\right) d Y_{D}^{2} .
\end{aligned}
$$

To obtain the limit as $\gamma \rightarrow \infty$ in the AdS case as in the absence of a charge, we can apply the lemma on distributions in $[60,85]$ :

$$
\lim _{v \rightarrow 1} \gamma f\left(\gamma^{2}\left(Y_{0}+v Y_{1}\right)^{2}\right)=\delta\left(Y_{0}+Y_{1}\right) \int f\left(x^{2}\right) d x .
$$

For the shape function $F_{D, \text { AdS }}\left(\bar{M}, \bar{Q}^{2}, Z\right)$, we obtain the formula

$$
F_{D, \mathrm{AdS}}\left(\bar{M}, \bar{Q}^{2}, Z\right)=F_{D, \mathrm{dS} / \operatorname{AdS}}(\bar{M}, Z)-\frac{\bar{Q}^{2}}{2 \bar{M}} F_{2 D-3, \operatorname{AdS}}(\bar{M}, Z)
$$

where

$$
F_{D, \mathrm{AdS}}(\bar{M}, Z)=2 \bar{M} a^{2} \int_{-\infty}^{\infty}\left[\frac{\left(a^{2}\left(-Z^{2}+x^{2}\right)+Z^{2}\left(x^{2}+Z^{2}\right)\right)}{\left(Z^{2}+x^{2}\right)^{2} \cdot\left(-a^{2}+x^{2}+Z^{2}\right)^{(D-1) / 2}}\right] d x .
$$

Here, $a^{2}=3 / \Lambda$.

We note that there are more subtleties in the case of the dS space $[34,86]$.

Open Access. This article is distributed under the terms of the Creative Commons Attribution License which permits any use, distribution and reproduction in any medium, provided the original author(s) and source are credited.

\section{References}

[1] J.M. Maldacena, The large- $N$ limit of superconformal field theories and supergravity, Int. J. Theor. Phys. 38 (1999) 1133 [Adv. Theor. Math. Phys. 2 (1998) 231] [hep-th/9711200] [INSPIRE].

[2] S. Gubser, I.R. Klebanov and A.M. Polyakov, Gauge theory correlators from noncritical string theory, Phys. Lett. B 428 (1998) 105 [hep-th/9802109] [INSPIRE].

[3] E. Witten, Anti-de Sitter space and holography, Adv. Theor. Math. Phys. 2 (1998) 253 [hep-th/9802150] [INSPIRE].

[4] G. Policastro, D. Son and A. Starinets, The Shear viscosity of strongly coupled $N=4$ supersymmetric Yang-Mills plasma, Phys. Rev. Lett. 87 (2001) 081601 [hep-th/0104066] [INSPIRE]. 
[5] J. Casalderrey-Solana, H. Liu, D. Mateos, K. Rajagopal and U.A. Wiedemann, Gauge/string duality, hot QCD and heavy ion collisions, arXiv:1101.0618 [INSPIRE].

[6] A. Bernamonti and R. Peschanski, Time-dependent AdS/CFT correspondence and the quark-gluon plasma, Nucl. Phys. Proc. Suppl. 216 (2011) 94 [arXiv:1102.0725] [InSPIRE].

[7] R.A. Janik, The Dynamics of quark-gluon plasma and AdS/CFT, Lect. Notes Phys. 828 (2011) 147 [arXiv:1003.3291] [INSPIRE].

[8] E. Shuryak, Why does the quark gluon plasma at RHIC behave as a nearly ideal fluid?, Prog. Part. Nucl. Phys. 53 (2004) 273 [hep-ph/0312227] [InSPIRE].

[9] E.V. Shuryak, What RHIC experiments and theory tell us about properties of quark-gluon plasma?, Nucl. Phys. A 750 (2005) 64 [hep-ph/0405066] [INSPIRE].

[10] U.W. Heinz, Thermalization at RHIC, AIP Conf. Proc. 739 (2005) 163 [nucl-th/0407067] [INSPIRE].

[11] R.A. Janik and R.B. Peschanski, Gauge/gravity duality and thermalization of a boost-invariant perfect fluid, Phys. Rev. D 74 (2006) 046007 [hep-th/0606149] [INSPIRE].

[12] R.A. Janik and R.B. Peschanski, Asymptotic perfect fluid dynamics as a consequence of AdS/CFT, Phys. Rev. D 73 (2006) 045013 [hep-th/0512162] [INSPIRE].

[13] S.S. Gubser, S.S. Pufu and A. Yarom, Entropy production in collisions of gravitational shock waves and of heavy ions, Phys. Rev. D 78 (2008) 066014 [arXiv:0805.1551] [INSPIRE].

[14] H. Nastase, On high energy scattering inside gravitational backgrounds, hep-th/0410124 [INSPIRE].

[15] H. Nastase, The RHIC fireball as a dual black hole, hep-th/0501068 [INSPIRE].

[16] E. Shuryak, S.-J. Sin and I. Zahed, A gravity dual of RHIC collisions, J. Korean Phys. Soc. 50 (2007) 384 [hep-th/0511199] [INSPIRE].

[17] A.J. Amsel, D. Marolf and A. Virmani, Collisions with black holes and deconfined plasmas, JHEP 04 (2008) 025 [arXiv: 0712.2221] [INSPIRE].

[18] D. Grumiller and P. Romatschke, On the collision of two shock waves in $A d S_{5}$, JHEP 08 (2008) 027 [arXiv:0803.3226] [INSPIRE].

[19] J.L. Albacete, Y.V. Kovchegov and A. Taliotis, Asymmetric collision of two shock waves in $A d S_{5}$, JHEP 05 (2009) 060 [arXiv: 0902.3046] [INSPIRE].

[20] A. Taliotis, Heavy ion collisions with transverse dynamics from evolving AdS geometries, JHEP 09 (2010) 102 [arXiv: 1004.3500] [INSPIRE].

[21] J.L. Albacete, Y.V. Kovchegov and A. Taliotis, Modeling heavy ion collisions in AdS/CFT, JHEP 07 (2008) 100 [arXiv:0805.2927] [INSPIRE].

[22] S. Khlebnikov, M. Kruczenski and G. Michalogiorgakis, Shock waves in strongly coupled plasmas, Phys. Rev. D 82 (2010) 125003 [arXiv: 1004.3803] [inSPIRE].

[23] S. Khlebnikov, M. Kruczenski and G. Michalogiorgakis, Shock waves in strongly coupled plasmas II, JHEP 07 (2011) 097 [arXiv:1105.1355] [INSPIRE].

[24] P.M. Chesler and L.G. Yaffe, Holography and colliding gravitational shock waves in asymptotically AdS $S_{5}$ spacetime, Phys. Rev. Lett. 106 (2011) 021601 [arXiv:1011.3562] [INSPIRE]. 
[25] B. Wu and P. Romatschke, Shock wave collisions in AdS $S_{5}$ : approximate numerical solutions, Int. J. Mod. Phys. C 22 (2011) 1317 [arXiv:1108.3715] [InSPIRE].

[26] M.P. Heller, R.A. Janik and P. Witaszczyk, The characteristics of thermalization of boost-invariant plasma from holography, arXiv:1103.3452 [INSPIRE].

[27] M.P. Heller, R.A. Janik and P. Witaszczyk, A numerical relativity approach to the initial value problem in asymptotically Anti-de Sitter spacetime for plasma thermalization - An ADM formulation, arXiv: 1203.0755 [INSPIRE].

[28] S.S. Gubser, S.S. Pufu and A. Yarom, Off-center collisions in AdS $S_{5}$ with applications to multiplicity estimates in heavy-ion collisions, JHEP 11 (2009) 050 [arXiv:0902.4062] [INSPIRE].

[29] S. Lin and E. Shuryak, Grazing collisions of gravitational shock waves and entropy production in heavy ion collision, Phys. Rev. D 79 (2009) 124015 [arXiv: 0902.1508] [INSPIRE].

[30] A. Duenas-Vidal and M.A. Vazquez-Mozo, Colliding AdS gravitational shock waves in various dimensions and holography, JHEP 07 (2010) 021 [arXiv: 1004.2609] [INSPIRE].

[31] L. Álvarez-Gaumé, C. Gomez, A. Sabio Vera, A. Tavanfar and M.A. Vazquez-Mozo, Critical formation of trapped surfaces in the collision of gravitational shock waves, JHEP 02 (2009) 009 [arXiv:0811.3969] [INSPIRE].

[32] Y.V. Kovchegov and S. Lin, Toward thermalization in heavy ion collisions at strong coupling, JHEP 03 (2010) 057 [arXiv: 0911.4707] [INSPIRE].

[33] S.S. Gubser and A. Nellore, Mimicking the QCD equation of state with a dual black hole, Phys. Rev. D 78 (2008) 086007 [arXiv:0804.0434] [INSPIRE].

[34] I.Y. Aref'eva, A. Bagrov and L. Joukovskaya, Critical trapped surfaces formation in the collision of ultrarelativistic charges in (A)dS, JHEP 03 (2010) 002 [arXiv:0909.1294] [INSPIRE].

[35] H. Yoshino and R.B. Mann, Black hole formation in the head-on collision of ultrarelativistic charges, Phys. Rev. D 74 (2006) 044003 [gr-qc/0605131] [INSPIRE].

[36] U. Gürsoy and E. Kiritsis, Exploring improved holographic theories for QCD: part I, JHEP 02 (2008) 032 [arXiv:0707.1324] [INSPIRE].

[37] U. Gürsoy, E. Kiritsis and F. Nitti, Exploring improved holographic theories for QCD: part II, JHEP 02 (2008) 019 [arXiv: 0707.1349] [INSPIRE].

[38] E. Kiritsis and A. Taliotis, Multiplicities from black-hole formation in heavy-ion collisions, JHEP 04 (2012) 065 [arXiv:1111.1931] [INSPIRE].

[39] I.Y. Aref'eva, Catalysis of black holes/wormholes formation in high energy collisions, Theor. Math. Phys. 161 (2009) 1647 [arXiv:0912.5481] [INSPIRE].

[40] D. Mateos, S. Matsuura, R.C. Myers and R.M. Thomson, Holographic phase transitions at finite chemical potential, JHEP 11 (2007) 085 [arXiv:0709.1225] [INSPIRE].

[41] S. Kobayashi, D. Mateos, S. Matsuura, R.C. Myers and R.M. Thomson, Holographic phase transitions at finite baryon density, JHEP 02 (2007) 016 [hep-th/0611099] [INSPIRE].

[42] K. Hashimoto, N. Iizuka and T. Oka, Rapid thermalization by baryon injection in gauge/gravity duality, Phys. Rev. D 84 (2011) 066005 [arXiv: 1012.4463] [INSPIRE].

[43] R.C. Myers, M.F. Paulos and A. Sinha, Holographic hydrodynamics with a chemical potential, JHEP 06 (2009) 006 [arXiv:0903.1596]. 
[44] S. Hands, T.J. Hollowood and J.C. Myers, QCD with chemical potential in a small hyperspherical box, JHEP 07 (2010) 086 [arXiv:1003.5813] [INSPIRE].

[45] N. Horigome and Y. Tanii, Holographic chiral phase transition with chemical potential, JHEP 01 (2007) 072 [hep-th/0608198] [INSPIRE].

[46] A. Parnachev, Holographic QCD with isospin chemical potential, JHEP 02 (2008) 062 [arXiv: 0708.3170] [INSPIRE].

[47] J. Erdmenger, M. Haack, M. Kaminski and A. Yarom, Fluid dynamics of R-charged black holes, JHEP 01 (2009) 055 [arXiv: 0809.2488] [INSPIRE].

[48] S. Hawking and D.N. Page, Thermodynamics of black holes in Anti-de Sitter space, Commun. Math. Phys. 87 (1983) 577 [INSPIRE].

[49] S. Gubser, I.R. Klebanov and A. Peet, Entropy and temperature of black 3-branes, Phys. Rev. D 54 (1996) 3915 [hep-th/9602135] [INSPIRE].

[50] L.D. Landau, On the multiparticle production in high-energy collisions, Izv. Akad. Nauk Ser. Fiz. 17 (1953) 51, english translation in Collected papers of L.D. Landau, D. ter Haar ed., Gordon and Breach, New-York U.S.A. (1968).

[51] J. Bjorken, Highly relativistic nucleus-nucleus collisions: the central rapidity region, Phys. Rev. D 27 (1983) 140 [inSPIRE].

[52] C. Fefferman and C.R. Graham, Conformal invariants, in Elie Cartan et les Mathématiques d'aujourd'hui, Astérisque 95 (1985).

[53] Y.V. Kovchegov, AdS/CFT applications to relativistic heavy ion collisions: a brief review, arXiv: 1112.5403 [INSPIRE].

[54] A. Chamblin, R. Emparan, C.V. Johnson and R.C. Myers, Charged AdS black holes and catastrophic holography, Phys. Rev. D 60 (1999) 064018 [hep-th/9902170] [INSPIRE].

[55] A. Chamblin, R. Emparan, C.V. Johnson and R.C. Myers, Holography, thermodynamics and fluctuations of charged AdS black holes, Phys. Rev. D 60 (1999) 104026 [hep-th/9904197] [INSPIRE].

[56] C.S. Peca and P. Lemos, Jose, Thermodynamics of Reissner-Nordstrom Anti-de Sitter black holes in the grand canonical ensemble, Phys. Rev. D 59 (1999) 124007 [gr-qc/9805004] [INSPIRE].

[57] M. Cvetič and S.S. Gubser, Phases of $R$ charged black holes, spinning branes and strongly coupled gauge theories, JHEP 04 (1999) 024 [hep-th/9902195] [INSPIRE].

[58] K. Skenderis, Lecture notes on holographic renormalization, Class. Quant. Grav. 19 (2002) 5849 [hep-th/0209067] [INSPIRE].

[59] I.Y. Aref'eva and I. Volovich, On the breaking of conformal symmetry in the AdS/CFT correspondence, Phys. Lett. B 433 (1998) 49 [hep-th/9804182] [INSPIRE].

[60] M. Hotta and M. Tanaka, Shock wave geometry with nonvanishing cosmological constant, Class. Quant. Grav. 10 (1993) 307 [INSPIRE].

[61] K. Sfetsos, On gravitational shock waves in curved space-times, Nucl. Phys. B 436 (1995) 721 [hep-th/9408169] [INSPIRE].

[62] G.T. Horowitz and N. Itzhaki, Black holes, shock waves and causality in the AdS/CFT correspondence, JHEP 02 (1999) 010 [hep-th/9901012] [INSPIRE]. 
[63] R. Emparan, Exact gravitational shock waves and Planckian scattering on branes, Phys. Rev. D 64 (2001) 024025 [hep-th/0104009] [InSPIRE].

[64] J. Podolsky and J. Griffiths, Impulsive waves in de Sitter and Anti-de Sitter space-times generated by null particles with an arbitrary multipole structure, Class. Quant. Grav. 15 (1998) 453 [gr-qc/9710049] [INSPIRE].

[65] I.Y. Aref'eva, A. Bagrov and E. Guseva, Critical formation of trapped surfaces in the collision of non-expanding gravitational shock waves in de Sitter space-time, JHEP 12 (2009) 009 [arXiv:0905.1087] [INSPIRE].

[66] R. Penrose, unpublished (1974).

[67] D.M. Eardley and S.B. Giddings, Classical black hole production in high-energy collisions, Phys. Rev. D 66 (2002) 044011 [gr-qc/0201034] [InSPIRE].

[68] N. Kaloper and J. Terning, How black holes form in high energy collisions, Int. J. Mod. Phys. D 17 (2008) 665 [Gen. Rel. Grav. 39 (2007) 1525] [arXiv:0705.0408] [INSPIRE].

[69] S.W. Hawking and G.R.E Ellis, The large scale structure of space-time, Cambridge University Press, Cambridge U.K. (1973).

[70] R.M. Wald, General relativity, The University of Chicago Press, Chicago U.S.A (1984).

[71] F. Karsch, Lattice QCD at high temperature and density, Lect. Notes Phys. 583 (2002) 209 [hep-lat/0106019] [INSPIRE].

[72] S. Klein and J. Nystrand, Exclusive vector meson production in relativistic heavy ion collisions, Phys. Rev. C 60 (1999) 014903 [hep-ph/9902259] [INSPIRE].

[73] STAR collaboration, J. Adams et al., Production of $e^{+} e^{-}$pairs accompanied by nuclear dissociation in ultra-peripheral heavy ion collision, Phys. Rev. C 70 (2004) 031902 [nucl-ex/0404012] [INSPIRE].

[74] S. Ochs and U.W. Heinz, Entropy production by resonance decays, Phys. Rev. C 54 (1996) 3199 [hep-ph/9606458] [INSPIRE].

[75] B. Müller and A. Schafer, Entropy creation in relativistic heavy ion collisions, Int. J. Mod. Phys. E 20 (2011) 2235 [arXiv: 1110.2378] [InSPIRE].

[76] B. Back et al., The significance of the fragmentation region in ultrarelativistic heavy ion collisions, Phys. Rev. Lett. 91 (2003) 052303 [nucl-ex/0210015] [INSPIRE].

[77] S. Lin and E. Shuryak, On the critical condition in gravitational shock wave collision and heavy ion collisions, Phys. Rev. D 83 (2011) 045025 [arXiv: 1011.1918] [INSPIRE].

[78] G. 't Hooft, Graviton dominance in ultrahigh-energy scattering, Phys. Lett. B 198 (1987) 61 [INSPIRE].

[79] G. 't Hooft, On the factorization of universal poles in a theory of gravitating point particles, Nucl. Phys. B 304 (1988) 867 [InSPIRE].

[80] S. Ghosh, S. Sarkar and J. Alam, Observing many body effects on lepton pair production from low mass enhancement and flow at RHIC and LHC energies, Eur. Phys. J. C 71 (2011) 1760 [arXiv:1009.1260] [INSPIRE].

[81] I.Y. Aref'eva, K. Viswanathan and I. Volovich, Planckian energy scattering, colliding plane gravitational waves and black hole creation, Nucl. Phys. B 452 (1995) 346 [Erratum ibid. B 462 (1996) 613] [hep-th/9412157] [INSPIRE]. 
[82] C. Lousto and N.G. Sanchez, The curved shock wave space-time of ultrarelativistic charged particles and their scattering, Int. J. Mod. Phys. A 5 (1990) 915 [InSPIRE].

[83] C. Lousto and N.G. Sanchez, Scattering processes at the Planck scale, gr-qc/9410041 [INSPIRE].

[84] M. Ortaggio, Ultrarelativistic boost of spinning and charged black rings, J. Phys. Conf. Ser. 33 (2006) 386 [gr-qc/0601093] [INSPIRE].

[85] P.C. Aichelburg and R.U. Sexl, On the gravitational field of a massless particle, Gen. Rel. Grav. 2 (1971) 303.

[86] I.Ya. Aref'eva, A.A. Bagrov and L.V. Joukovskaya, Some aspects of the application of generalized functions to the analysis of gravitational shock waves in general relativity, Alg. $i$ Anal. 22 (2010) 3 [St. Petersburg Math. J. 22 (2011) 337]. 